\title{
Article
}

\section{Genetic characterization of $Y$ - chromosomal STRs in Hazara ethnic group of Pakistan and confirmation of DYS448 null allele}

Adnan, Atif, Rakha, Allah, Kasim, Kadirya, Noor, Anam, Nazir, Shahid, Hadi, Ss and Pang, Hao

Available at http://clok.uclan.ac.uk/24842/

Adnan, Atif, Rakha, Allah, Kasim, Kadirya, Noor, Anam, Nazir, Shahid, Hadi, Ss ORCID: 0000-0002-2994-3083 and Pang, Hao (2019) Genetic characterization of Y-chromosomal STRs in Hazara ethnic group of Pakistan and confirmation of DYS448 null allele. International Journal of Legal Medicine, 133 (3). pp. 789793. ISSN 0937-9827

It is advisable to refer to the publisher's version if you intend to cite from the work. http://dx.doi.org/10.1007/s00414-018-1962-x

For more information about UCLan's research in this area go to http://www.uclan.ac.uk/researchgroups/ and search for < name of research Group>.

For information about Research generally at UCLan please go to http://www.uclan.ac.uk/research/

All outputs in CLoK are protected by Intellectual Property Rights law, including Copyright law. Copyright, IPR and Moral Rights for the works on this site are retained by the individual authors and/or other copyright owners. Terms and conditions for use of this material are defined in the policies page. 


\section{Genetic characterization of Y-chromosomal STR in Hazara ethnic group of Pakistan and}

confirmation of DYS448 null allele

Atif Adnan ${ }^{1}$, Allah Rakha ${ }^{2}$, Anam Noor ${ }^{2}$, Shahid Nazir ${ }^{2}$, Jinfeng Xuan ${ }^{1}$, Jiaxin Xing ${ }^{1}$, Sibte

Hadi $^{3}$ and Hao Pang ${ }^{1^{*}}$

1 Department of Forensic Genetics and Biology, School of Forensic Medicine, China Medical University, Shenyang 110122, P.R. China

2 Department of Forensic Sciences, University of Health Sciences Lahore, 54000, Pakistan

3 University of Central Lancashire, School of Forensic and Investigative Sciences, Preston, UK

*Corresponding author:

Hao Pang (hpang@cmu.edu.cn) 


\section{ABSTRACT:}

Pakistan harbors 18 major ethnic groups and Hazara is one of the distinct but smaller groups comprising $0.090 \%$ of the total population of Pakistan. Hazara individuals have typical Mongolian facial features and they claim to be descendants of Genghis Khan's army in the first quarter of the thirteenth century AD. Previous study based on limited number of Y STRs with limited number of samples showed that Hazaras are descendants of Mongols. In this study, we genotyped 198 unrelated males (153 Hazara, 15 Baluchi and 30 Mongols) living in Quetta, Baluchistan, Pakistan and Inner Mongolia, China respectively, for a total of $26(n=153)$ to 29 (n=92) Y-chromosomal STR loci. 140 unique haplotypes were developed for Hazara population using the PowerPlex Y23 loci. The Y-STR locus showed a genetic diversity ranging from 0.2384 to 0.7918 , and an overall discrimination capacity (DC) of $91.5 \%$. The Hazara population samples were profiled for three additional Y-STRs (DYS388, DYS449 and DYS460), which increased the number of unique haplotypes to 144 while the DC increased to $94.11 \%$ in Hazara Population of Pakistan. Interestingly null alleles were observed at DYS448 with a specific mutation patterns in 25 individuals of Hazara population. The Hazaras showed significant differences from other local populations of Pakistan as well as neighboring populations, but had considerable genetic affinities to Kazakhs and Mongols. There was a lack of data for Hazara population of Pakistan and our results thus contribute to understanding the potential forensic usefulness of the $26 \mathrm{Y}$ STRs studied and also shed light on the population history of Pakistani Hazara population. 


\section{INTRODUCTION}

Pakistan harbors more than 18 major ethnic groups, over 210 million people

(http://www.pbscensus.gov.pk). The genetic legacy of many of the ethnic groups in Pakistan has not been studied in depth and data for these ethnic groups is scarce[1]. The origin of Hazara population is disputed. The Hazara could be of Turko-Mongol ancestry, and theorized to be the descendants of an occupying army left in Afghanistan by Genghis Khan[2]. The Hazara population speaks Persian with some Mongolian words. An earlier study done on a limited number of samples $(n=33)$ had shown them to be closer to populations in Mongolia1 and the star shape phylogenetic tree of this population using Y-STR haplotypes was suggestive of their Mongolian roots[3].

The human Y chromosome markers are remarkably variable, and significantly used for population lineage and human migration studies; this primarily has been due to their ability to differentiate different paternal lineages[4-6]. Molecular population genetics studies have shown that many structural variants exist within human Y chromosome, includes deletions[7-9], duplications[10-12], and inversions[11-15]. Null alleles are known to occur with any PCRbased STR typing system. These could be either due to deletions within the target region or primer binding site mutations[16,17]. DYS448 is located adjacent to the azoospermia factor c $(\mathrm{AZFc})$ region, which is considered important in spermatogenesis and made up of " ampliconic", repeats that act as substrates for nonallelic homologous recombination (NAHR). NAHR might delete longer chunks of the Y chromosome which included DYS448[18].

Short tandem repeats (STRs) can reveal more recent events in population history than single nucleotide polymorphisms (SNPs), because of their higher mutability and allelic polymorphism. 
Y-STRs Plays vital role in forensic genetics, particularly for sexual offences analysis; paternity testing and investigations related missing persons [19-22]. Specifically Y STR analysis can detect low quantity of male DNA within high background female DNA as typically challenged within cases of sexual assault[23].

In this study we have studied Pakistani Hazara population using extended sets of Y STRs to determine genetic diversity and to explore their history. 25 null alleles were observed at DYS448 in 153 Hazara male samples. This data has defined the Hazara population better and will be an addition to the Y STR haplotype reference database (YHRD).

\section{MATERIALS AND METHODS}

Samples used in the study:

Blood samples were collected from, unrelated-One hundred and fifty three unrelated 153-Hazara \& 15 Baluchi males residing in Quetta, Baluchistan, Pakistan and 30 Mongols living in Inner Mongolia, P.R. China volunteered to provide blood samples. The samples were collected as blood stains on TIANamp blood Spots DNA Kit (TIANGEN BIOTECH BEIJING CO., LTD). Informed consent was obtained from all participants before obtaining the sample.All participants gave their informed consent either orally (in case they could not write) or in writing, after the study aims and procedures were explained them carefully. The study was approved by the-The thical review committee of China Medical University, Shenyang, Liaoning Province, P.R. China and in accordance with the standards of the Declaration of Helsinkiapproved the project and obtaining of samples.

DNA extraction: 
Genomic DNAs were extracted from blood stains by xxxxxxx using TIANamp blood Spots DNA Kit (TIANGEN BIOTECH BEIJNG CO., LTD) according to the manufacturer's instructions_-(TIANGEN BIOTECH BEIJING CO., LTD).

\section{PCR amplification and Y-STR typing:}

26 Y STR loci (which contains PowerPlex Y23 loci and three additional Y-STRs DYS388, DYS449 and DYS460) were amplified using the Goldeneye ${ }^{\circledR} 26 Y$ system (PEOPLESPOT R\&D, China) as described elsewhere[24]. The 2800M (Promega Corporation, USA) was used as positive control in all batches. All PCR batches included a PCR negative control. The PCR products were separated and detected by capillary electrophoresis on an ABI 3500 Genetic Analyzer (Applied Biosystems, USA). The genotyping results were analyzed using GeneMapper ID v3.2 (Applied Biosystems, USA).

\section{Identification of DYS448 deletions}

Yfiler $^{\mathrm{TM}}$ Plus PCR Amplification Kit (Applied Biosystems, USA) and Microreader ${ }^{\mathrm{TM}}$ 29Y Direct ID System (DYS393, DYS570, DYS19, DYS392, DYS549, Y-GATAH4, DYF387S1, DYS460, DYS458, DYS481, DYS635, DYS448, DYS533, DYS627, DYS456, DYS389I, DYS390, DYS389II, DYS438, DYS576, DYS449, DYS391, DYS439, DYS437, DYS385a/b, DYS643, DYS518) kits were used to confirm the null alleles at DYS448 according to the manufacturer's protocol. 47 samples were used for this exercise which generated haplotypes comprising of Yfiler ${ }^{\text {TM }}$ Plus kit and 30 Y STRs.

DYS 448 Sequencing

25 Hazara samples showed DYS448 null alleles (detected as allelic dropouts) in three Y STR kits (GoldenEye Y26, Microreader ${ }^{\mathrm{TM}}$ Y29 and Yfiler ${ }^{\mathrm{TM}}$ Plus). The region was amplified using a
Commented [SH1]: Pl say how this was done e. a $1 \mathrm{~mm}$ punch was taken from the stain and was heated at $56 \mathrm{C}$ for 30 mins and so on. 
nested PCR to reduce unspecific PCR products. First-round PCR was performed with the forward primers 5'-AGAAGAATGGATGATGAGAGATCTG-3' and the reverse primer 5'CATCTCCTGGAGTCAGACAGTAATC-3', resulting in an 1193bp PCR product. Secondround of PCR was performed using forward primer

5'-GCATGCCAACAACAAGGATAT-3' and the reverse primer 5'-GTTTCCCTGACTCCATTAGCTG-3' resulting in approximately 500bp product. Bothrounds of PCR reaction was carried out in a $20 \mu \mathrm{l}$ volume containing $2 \mu \mathrm{l}$ of $10 \times$ LA PCR $^{\mathrm{TM}}$ Buffer II (Mg ${ }^{2+}$ Plus) (TaKaRa Bio Inc., Dalian, China.), $2 \mu \mathrm{l}$ dNTP Mixture (2.5 mM each) (TaKaRa), $0.25 \mu \mathrm{l} \mathrm{TaKaRa} \mathrm{LA} \mathrm{Taq}{ }^{\text {TM }}$ (5 units/ $\left.\mu \mathrm{l}\right)$ (TaKaRa), $14.75 \mu l \mathrm{ddH}_{2} \mathrm{O}$ and $1 \mu \mathrm{l}$ of genomic DNA. In first round $1 \mu \mathrm{l}$ of DNA was used and in second round we $1 \mu \mathrm{l}$ of $20 \times$ to $100 \times$ PCR products of first round, were used. Samples were amplified in a GeneAmp 9700 PCR machine (Applied Biosystems, Foster City, CA, USA) under following conditions for both rounds of amplification: one denaturation cycle of $94{ }^{\circ} \mathrm{C}$ for $1 \mathrm{~min} ; 35$ amplification cycles of $94{ }^{\circ} \mathrm{C}$ for 30 s, $65{ }^{\circ} \mathrm{C}$ for $30 \mathrm{~s}$ and $72^{\circ} \mathrm{C}$ for $1 \mathrm{~min}$; and one full extension cycle of $72{ }^{\circ} \mathrm{C}$ for $5 \mathrm{~min}$. The PCR products around $500 \mathrm{bp}$ were purified by centrifugation by using a Suprec-02 tube (Takara) and then were sequenced directly with second round primers. The Sequencing reaction was carried out in $20 \mu \mathrm{l}$ reaction volume, which contains $1 \mu$ l of PCR product, $8 \mu$ l of BigDye $(2.5 \times)$, each primer (F and R) $(3.2 \mathrm{mM}) 1 \mu \mathrm{l}$ and $\mathrm{dH}_{2} \mathrm{O} 10 \mu \mathrm{l}$ under following conditions $96{ }^{\circ} \mathrm{C}$ for $1 \mathrm{~min} ; 25$ amplification cycles of $96{ }^{\circ} \mathrm{C}$ for $10 \mathrm{sec}, 50{ }^{\circ} \mathrm{C}$ for $5 \mathrm{sec}$; and one full extension of $60{ }^{\circ} \mathrm{C}$ for 4 min. Then purified PCR products were used for sequencing by capillary electrophoresis using 3500 Genetic Analyzer (Thermo Fisher Scientific) according to manufacturer's manual. 


\section{Statistical analyses:}

Allelic frequencies were developed through simple count method. computed using the method of gene counting. Gene diversity (GD) or haplotype diversity (HD) was calculated by Nei's formula was used to calculate Gene diversity (GD) which is equivalent to haplotype diversity (HD) for Y STRs [25]. Y STR data generated using Goldeneye ${ }^{\circledR} 26 \mathrm{Y}$ kit and the Yfiler ${ }^{\mathrm{TM}}$ Plus kit allowed the development of Hhaplotype diversitiesy (HD) wereas calculatedestimated for the 26/27 Y STR loci included in these kits and also the haplotype diversities for the 9 Y STR loci of minimal (9 loci) haplotype, 11 loci of; extended (11 loci); haplotype, Powerplex Y (12 loci); -Y

(17 loci) and ; Powerplex Y 23 (23 loci),-Goldeneye (B) 26Y kit, Y filerTM Plus (27 loci) and a An extended haplotype comprising of Y filer ${ }^{\mathrm{TM}}$ Plus with three additional loci (30 loci) was also developed and haplotype diversity calculated. Discrimination capacity (DC) was calculated as the ratio of unique haplotypes in the sample. Match probabilities (MP) were calculated as $\Sigma \mathrm{P}^{2}$, where $\mathrm{P} i$ is the frequency of the $i$-th haplotype. Genetic distances between Hazara population and reference population analysis of other world populations were calculated using molecular variance (AMOVA) and multidimensional scaling (MDS). Both these analyses were-that exploits variation ameng pepulations were performed using YHRD online tools (http://www.yhrd.org) based on pairwise Rst values.

Phylogenetic analysis:

A neighbor-joining phylogenetic tree was constructed for the Hazara and the reference populations based on a distance matrix of Rst using the Mega7 software[26]. We also predicted Y-SNP haplogroups in the samples from Y STR haplotypes using the Y-DNA Haplogroup Predictor NEVGEN (http://www.nevgen.org).

The median joining network 
Using the program Network 4.1.1.2.( http://www.fluxus-engineering.com/sharenet.htm), median joining network was constructed from data of Hazara population for 23 Y STRs (DYS19, DYS389II-I, DYS390, DYS391, DYS392, DYS393, DYS437, DYS438, DYS439, DYS456, DYS458, DYS635, Y_GATA_H4, DYS576, DYS570, DYS481, DYS533, DYS549, DYS643, DYS460, DYS449 and DYS388).

\section{Linear discriminant analysis and Correlation}

Linear discriminant analysis (LDA) was performed on Hazara, Central Asia, East Asia; Middle East and South West Asian samples using R program[27] and correlation between STRs were calculated using XLSTAT (http://www.xlstat.com/en/). The multi-copy marker like (DYS385ab) and markers that have null alleles or duplication variants in the Hazara population or any of the reference populations were excluded from the analysis. For DYS389I and DYS389II, we have subtracted DYS389I from DYS389II and used DYS389II-I for analysis.

\section{RESULTS AND DISCUSSION}

We successfully obtained-Y-STR haplotypes from 153 male individuals in Hazara population were developed. The data showedwith 25 null alleles for DYS448. 144 (out of 153) different haplotypes were observed for 26 Y STRs while $47(n=47)$ haplotypes observed for 30 Y STRs (Supplementary material Table S1A). Allelic frequencies and GD values of various sets of YSTR loci in Hazara individuals were calculated (Table 1). 199 alleles were observed for all $30 \mathrm{Y}$ STRs. The allelic frequencies of single-copy STR loci ranged from 0.0065 to 0.8627 , while the frequencies of genotypes of multi-copy STR loci ranged from 0.0065 to 0.4183 . DYS385 (0.960), DYS387S1 (0.813) and DYS627 (0.792) revealed highest GD values, revealing a high level of genetic polymorphism in the Hazara population of Pakistan. Hazara population of 
Pakistan showed almost same allelic frequencies on minimum haplotype (MH) STRs with Mongols of Inner Mongolia and Kazak population of Kazakhstan, while have differences on GD values. However, since the focus of this study was on Hazara population, we did not aim to discuss Baluchi and Mongol population in detail due to small data set but the genotype data for Baluchi and Mongol populations was tabulated (Supplementary material Table S1B and Supplementary material Table S1C). Allelic frequencies and GD values of Baluchi and Mongols were also calculated (Table 2 and Table 3 ).

\section{HAPLOTYPE DIVERSITY}

The increase in the number of Y STR loci enhanced haplotype diversity for the set of 153 male samples. This ranged from 0.93 to almost 1 as the number of loci increased from 9 minimum haplotype (MH) STRs loci to 30 extended STRs loci (Table 4). Haplotype data already made accessible via YHRD under accession number YC000340. The increase of haplotype diversity was small between the Powerplex Y 23 and Goldeneye ${ }^{\circledR} 26$ Y kit $(0.9981 \& 0.9985$ respectively). This increase in haplotype diversity was parallel to the increase in the number of unique haplotypes which ranged from 75-97\% (Table 4). In this set of 153 male samples the Goldeneye ${ }^{\circledR} 26$ Y kit generated 144 haplotypes among which 140 were unique haplotypes whereas if the PowerPlex Y23 kit loci were considered there were 140 haplotypes with only 132 being unique. This substantial increase in the proportion of unique haplotypes revealed the greater power of discrimination of Goldeneye 26 Y STR loci in the Hazaras. This trend was also noted for the smaller set of 45 samples for the Baluchi and Mongol populations which were profiled using Yfiler ${ }^{\mathrm{TM}}$ plus and 2 additional Y STR loci as well (Table 5 and 6). Thus both, Goldeneye ${ }^{\circledR} 26 \mathrm{Y}$ and Yfiler ${ }^{\mathrm{TM}}$ systems can potentially be used for forensic casework and population genetic studies for smaller ethnic groups like the Hazaras in Pakistan. 


\section{GENETIC RELATIONSHIP OF THE HAZARAS WITH MONGOL POPULATION}

Previous studies based on uni-parental markers suggesting that Hazaras are the male descendants of Genghis Khan, or other Mongol populations[1,28]. In order to study the genetic relationship of the Hazara population, the genotypes of the sample set were used to calculate the Rst values for the Harazas (Supplementary Table S1A). Then the multidimensional scaling analysis (MDS) analysis was performed among the Hazara population of Pakistan and 27 other reference populations based on Rst values (Table 7 and Figure 1). Hazara population was located closest to previously studied Hazara population (Rst -0.004), Kohistani population (Rst -0.0049) and Kazakh population (Rst -0.0048) of Kazakhstan. The Khalkh population (Rst 0.0117) of Central Mongolia and Mongolian population (Rst 0.0158) of Ulaanbaatar, Mongolia also appeared to be clustered with the Hazara population. It was established earlier that at least third to half of Hazara chromosomes were of East Asian origin[29]. In current study linear discrimination analysis (LDA) analysis was performed (Figure 2), which showed that Hazara not even have East Asian origin there is also influence of Central Asian populations. LDA was also performed between Hazara population of Pakistan and five other populations including Hazara population of Afghanistan, Kazakh and Uyghur population of Xinjiang, Mongol population from Inner Mongolia China (Current Study) and Baluchi population from Pakistan (Current Study).

Evolutionary relationships between the Hazara population of Pakistan and these five populations were also inferred from the Neighbor-joining tree based on the Fst values (Figure 3). LDA plot showed the association between 4 populations (Hazara Pakistan, Hazara Afghanistan, Kazakh of Xinjiang and Mongol of Inner Mongolia China) while Uyghur was placed on the right side of LDA and Baluchi population was placed on left side (Figure 4). 
Evolutionary relationships between the Hazara population of Pakistan and other reference populations were inferred from the Neighbor-joining tree based on the $\mathrm{R}_{S T}$ values (Figure 5). The similarities between the Hazara and the Kazak population were consistent with historical records, which established that present Kazak population was an admixture of Central Asian, Mongolian, Uyghur and other populations formed around the 13th century[30].

\section{INFERENCE OF ANCESTRY BASED ON Y STRS}

Y chromosomal and mtDNA variations are not interrupted by recombination, so is conserved in both lineages and associates strongly with continental regions. The Hazara samples exhibited two main haplogroups M217 in 71 samples and M420 in 21 samples (147 out of 153 Hazara

Pakistan samples used for this analysis). M217 is found at high frequencies among Central Asian populations (Mongolians), indigenous Siberians (Kazakhs) and M420 which is distributed in a large region in Eurasia, extending from Scandinavia and Central Europe to southern Siberia and South Asia[3,31,32]. These results support the view that Hazaras of Pakistan may have Mongol[1,32] and Kazakh origin. The median joining network based on 23 Y STR loci shows the extent of variation within Hazara population (Figure 6).

LDA was performed on the Hazara population of Pakistan, Central Asian, Middle Eastern, south west Asian and East Asian population samples to explore the potential for markers that are ancestry-informative. Figure 2A shows all individual samples plotted on the two LDA factors (F1 and F2). The first factor (DYS635) explained the majority (46.52\%) of the variation while second factor (DYS389II) explained the minority (34.79\%) of variations. The markers DYS635 and DYS389II had the largest correlation coefficient $(0.7064$ and 0.5188$)$ with the first and second factor, respectively (Figure 2B). LDA Plot showed the association of Hazara population of Pakistan with East and Central Asian populations. 


\section{PHYSICAL CHARACTERIZATION OF DYS448 DELETIONS}

Most of human Y chromosomes have a single DYS448 allele, we observed that some have carried null alleles. Null alleles usually can occur due to deletions within the target region or primer binding sites or by primer binding site mutations that destabilize hybridization of at least one of the primers flanking the target region[33-36]. By using GoldenEye Y26 system null types at DYS448 were observed from 26 individuals (Figure 7) in Hazara population of Pakistan. The phenomena of null allele was previously reported, in which other commercial kits were used[3742]. Current population study represents the high frequencies of null allele at DYS448 when compared with previously reported population to date (Table 8). When we used Microreader Y29 system the results were same as with GoldenEye Y26. Then finally we used Yfiler ${ }^{\mathrm{TM}}$ Plus kit which also showed null alleles in these 25 individuals (out of 26).

One individual who shown allele 20 at the DYS448 locus was selected and sequenced to serve as a reference for alignment. All samples who showed null allele at DYS448 (25 samples), also showed a specific pattern of deletion which might be specific to Hazara population of Pakistan

(Table 9). To confirm this phenomenon more studies are required on Hazara population of Pakistan and Afghanistan. Frequency of null allele at DYS448 is more common in Asia more specifically in East and Central Asia when it compared to rest of the world[18,38]. Commercial companies should give special attention while designing DYS448 primers.

\section{CONCLUDING REMARKS}

In current study we have genotyped 153 Hazara individuals for 26 Y STRs, and 45 Baluchi \& Mongol individuals for 29 Y STRs. The genetic variation in Hazara population and its comparison to several relevant populations were done using various statistical tests. This showed 
that the Hazara population has close affinity to the Central Asian and Mongol populations. In previous study[3] star-cluster profile was 10-16-25-10-11-13-14-12-11-11-11-12-8-10-10, for the loci DYS389I-DYS389b-DYS390-DYS391-DYS392-DYS393-DYS388-DYS425-DYS426DYS434-DYS435-DYS436-DYS437-DYS438-DYS439. In current study most frequent haplotype was 15-13-29-24-10-11-13-14-11-12 for loci DYS19-DYS389I-DYS389II-DYS390DYS391-DYS392-DYS393-DYS437-DYS438-DYS439 found in 43 individuals while 14-13-2924-8-11-13-14-11-11 found in 9 individuals and 15-13-29-24-11-11-13-14-11-12 found in 8 individuals. These haplotypes are most frequent haplotype in Mongols and Kazakhs[43]. Allelic Frequencies on these 10 Y STRs are almost similar to Kazak43 population of Kazakhstan Central Asia and somehow similar to Mongol population of Inner Mongolia. Our results contributed towards deciphering the origin of Hazara population of Pakistan and supported the view that they had Kazakh and Mongol origins. The forensic parameter calculations showed high discriminatory power with potential applications for forensic casework. We detected 25 null alleles at the locus DYS448 that equated to $16 \%$ of null alleles at this locus. This was the highest percentage of DYS448 null alleles in a population to date. The null allele sequencing results showed a specific deletion patterns, which need to be further, investigated in the Afghani Hazaras and also Mongol populations for assessing the impact of the sequence.

\section{ACKNOWLEDGEMENTS}

We are very grateful to the volunteers in our study in particular Fengrui Li, Mannis Van Ovan, Muhammad Farhan, Ammar Sabir Cheema, Xiaoni Zhan and Fatima. This project is supported by the National Natural Science Foundation of P. R. China (NSFC, No. 81471826).

\section{AUTHOR CONTRIBUTIONS}


H.P. and A.A. developed the idea and designed the experimental approach; A.A., A.R., A.N. and S.N. had collected the samples, A.A. performed the experiments, supported by J.X., Jf. X.; A.A. analyzed the data, A.A. wrote the initial manuscript, which is modified by A.A., H.P., S.H., A.R. and A.N. All authors reviewed the manuscript.

\section{COMPETING INTERESTS}

The authors declare no competing interests.

\section{FIGURES}

\section{Figure 1:}

Two-dimensional plot from multi-dimensional scaling analysis of Rst-values based on Yfiler haplotypes for Hazara population of Pakistan with reference to 27 other populations.

\section{Figure 2:}

A) LDA Analysis between Hazara, Central Asia, South Asia, Russia and East Asian populations.

B) Correlation coefficient of different Y STR markers and the two LDA factors

\section{Figure 3:}


A) LDA Analysis between Hazara Pakistan, Hazara Afghanistan, Baluchi Pakistan, Kazakh Xinjiang, Uyghur Xinjiang and Mongolian population of Inner Mongolia China populations.

B) Correlation coefficient of different Y STR markers and the two LDA factors

\section{Figure 4:}

Neighbour-joining phylogenetic tree of the Hazara population and the Central Asia, South Asia, Russia and East Asian populations based on a distance matrix of Rst.

\section{Figure 5:}

Neighbour-joining phylogenetic tree of the Hazara population and the Central Asia, South Asia, Russia and East Asian populations based on a distance matrix of Fst.

Figure 6:

The median joining network of Hazara population of Pakistan based on 23 Y STR

\section{Figure 7:}

Null types at DYS448 were observed from 25 to 26 individuals based on 3 different kits (GoldenEye Y 26, Microreader Y 29 and Yfiler Plus)

\section{TABLES:}

\section{Table 1:}

Allele Frequencies of Hazara population of Pakistan on 30 Y-STRs

\section{Table 2:}

Allelic frequencies of Baluchi population of Pakistan based on 29 Y STRs

\section{Table 3:}

Allelic frequencies of Mongol population of Inner Mongolia, China based on 29 Y STRs

\section{Table 4:}


Forensic statistical parameters in Hazara population of Pakistan on 8 levels

\section{Table 5:}

Forensic statistical parameters in Baluchi population of Pakistan on 8 levels

\section{Table 6:}

Forensic statistical parameters in Mongol population of Inner Mongolia China on 8 levels

\section{Table 7:}

Pairwise Rst values between Hazara Population of Pakistan and other 27 reference populations

\section{Table 8:}

Frequencies of null allele at DYS448 in various ethnic groups across continents

\section{Table 9:}

Sequence in the relevant flanking and repeat region of the DYS448 locus for normal and null alleles

\section{SUPPLIMENTRY TABLES}

\section{Table S1A}

The haplotype distributions and haplotype frequencies of Hazara population from Pakistan $(n=153)$.

\section{Table S1B}

The haplotype distributions and haplotype frequencies of Baluchi population from Pakistan $(n=15)$.

\section{Table S1C}


The haplotype distributions and haplotype frequencies of Mongol population from Inner Mongolia China $(\mathrm{n}=30)$.

Tables:

Table 1: Allele Frequencies of Hazara population of Pakistan on 30 Y-STRs

\begin{tabular}{|c|c|c|c|c|c|c|c|c|c|c|c|c|c|}
\hline Allele & DYS19 & Allele & DYS518 & Allele & DYS533 & Allele & DYS390 & Allele & DYS439 & Allele & DYS643 & Allele & DYS576 \\
\hline 12 & 0.0065 & 37 & 0.0638 & 10 & 0.0131 & 19 & 0.0196 & 8 & 0.0065 & 8 & 0.0196 & 15 & 0.0065 \\
\hline 13 & 0.0458 & 38 & 0.0638 & 11 & 0.1177 & 22 & 0.0458 & 9 & 0.0261 & 9 & 0.0784 & 16 & 0.1242 \\
\hline 14 & 0.2157 & 39 & 0.6170 & 12 & 0.6013 & 23 & 0.0850 & 10 & 0.1307 & 10 & 0.7582 & 17 & 0.5621 \\
\hline 15 & 0.6275 & 40 & 0.1489 & 13 & 0.0654 & 24 & 0.6732 & 11 & 0.1961 & 11 & 0.0850 & 18 & 0.1961 \\
\hline 16 & 0.0784 & 41 & 0.0851 & 14 & 0.1438 & 25 & 0.1569 & 12 & 0.5882 & 12 & 0.0523 & 19 & 0.0850 \\
\hline 17 & 0.0261 & 42 & 0.0213 & 15 & 0.0588 & 27 & 0.0196 & 13 & 0.0523 & 13 & 0.0065 & 20 & 0.0261 \\
\hline $\mathrm{AC}$ & 6 & $\mathrm{AC}$ & 6 & $\mathrm{AC}$ & 6 & $\mathrm{AC}$ & 6 & $\mathrm{AC}$ & 6 & $\mathrm{AC}$ & 6 & AC & 6 \\
\hline GD & 0.5544 & GD & 0.5939 & GD & 0.5999 & GD & 0.5155 & GD & 0.5989 & GD & 0.4113 & GD & 0.6263 \\
\hline Allele & DYS388 & Allele & DYS389I & Allele & DYS389II & Allele & DYS438 & Allele & DYS391 & Allele & DYS549 & Allele & DYS456 \\
\hline 12 & 0.2418 & 12 & 0.0588 & 28 & 0.0392 & 9 & 0.0588 & 8 & 0.1307 & 10 & 0.0327 & 13 & 0.0523 \\
\hline 13 & 0.5490 & 13 & 0.8235 & 29 & 0.7451 & 10 & 0.1699 & 9 & 0.0327 & 11 & 0.1765 & 14 & 0.0654 \\
\hline 14 & 0.1046 & 14 & 0.0915 & 30 & 0.1111 & 11 & 0.7059 & 10 & 0.6078 & 12 & 0.7124 & 15 & 0.2484 \\
\hline 15 & 0.0784 & 11,14 & 0.0131 & 31 & 0.0719 & 9,11 & 0.0065 & 11 & 0.2222 & 13 & 0.0719 & 16 & 0.1111 \\
\hline 16 & 0.0261 & 15 & 0.0131 & 32 & 0.0327 & 12 & 0.0588 & 12 & 0.0065 & 14 & 0.0065 & 17 & 0.5229 \\
\hline $\mathrm{AC}$ & 5 & $\mathrm{AC}$ & 5 & $\mathrm{AC}$ & 5 & $\mathrm{AC}$ & 5 & $\mathrm{AC}$ & 5 & $\mathrm{AC}$ & 5 & $\mathrm{AC}$ & 5 \\
\hline GD & 0.6264 & GD & 0.3117 & GD & 0.4275 & GD & 0.4690 & GD & 0.5667 & GD & 0.4580 & GD & 0.6498 \\
\hline
\end{tabular}




\begin{tabular}{|c|c|c|c|c|c|c|c|c|c|c|c|c|c|}
\hline Allele & DYS460 & Allele & DYS392 & Allele & DYS570 & Allele & GATA_H4 & Allele & DYS393 & Allele & DYS437 & Allele & DYS448 \\
\hline 7 & 0.0065 & 10 & 0.0065 & 15 & 0.0131 & 10 & 0.0196 & 12 & 0.1242 & 14 & 0.8628 & Null & 0.1699 \\
\hline 9 & 0.6013 & 11 & 0.8431 & 16 & 0.1046 & 11 & 0.6797 & 13 & 0.8562 & 15 & 0.1373 & 18 & 0.0065 \\
\hline 10 & 0.1307 & 12 & 0.0261 & 17 & 0.0850 & 12 & 0.1895 & 14 & 0.0196 & $\mathrm{AC}$ & 2 & 19 & 0.3072 \\
\hline 11 & 0.2418 & 13 & 0.0850 & 18 & 0.1503 & 13 & 0.1111 & $\mathrm{AC}$ & 3 & GD & 0.2384 & 20 & 0.2614 \\
\hline 12 & 0.0196 & 14 & 0.0131 & 19 & 0.4837 & $\mathrm{AC}$ & 4 & GD & 0.2528 & & & 21 & 0.1307 \\
\hline $\mathrm{AC}$ & 5 & 15 & 0.0196 & 20 & 0.1438 & GD & 0.4925 & & & & & 22 & 0.1111 \\
\hline \multirow[t]{4}{*}{ GD } & 0.5661 & 16 & 0.0065 & 21 & 0.0196 & & & & & & & 23 & 0.0065 \\
\hline & & $\mathrm{AC}$ & 7 & $\mathrm{AC}$ & 7 & & & & & & & 24 & 0.0065 \\
\hline & & GD & 0.2824 & GD & 0.7087 & & & & & & & $\mathrm{AC}$ & 8 \\
\hline & & & & & & & & & & & & GD & 0.7840 \\
\hline Allele & DYS635 & Allele & DYS449 & Allele & DYS458 & Allele & DYS481 & Allele & DYS627 & Allele & DYS387S1 & Allele & DYS385a,b \\
\hline 18 & 0.0131 & 26 & 0.0261 & 12 & 0.0065 & 20 & 0.0131 & 16 & 0.0213 & 35,35 & 0.0213 & 10,11 & 0.0065 \\
\hline 19 & 0.0065 & 27 & 0.0654 & 14 & 0.0327 & 22 & 0.0980 & 17 & 0.0638 & 36,36 & 0.0638 & 10,12 & 0.0065 \\
\hline 20 & 0.1895 & 28 & 0.5686 & 15 & 0.0784 & 23 & 0.1634 & 18 & 0.0638 & 36,37 & 0.0213 & 10,13 & 0.0065 \\
\hline 21 & 0.4837 & 29 & 0.0654 & 16 & 0.2418 & 24 & 0.0523 & 19 & 0.0213 & $36,38,40$ & 0.0213 & 11,11 & 0.0131 \\
\hline 22 & 0.1111 & 30 & 0.0980 & 17 & 0.4967 & 25 & 0.0523 & 20 & 0.3830 & $36,38,39$ & 0.0213 & 11,12 & 0.0065 \\
\hline 23 & 0.1503 & 31 & 0.0327 & 18 & 0.0915 & 26 & 0.0458 & 21 & 0.2128 & 36,39 & 0.0213 & 11,13 & 0.0065 \\
\hline 24 & 0.0392 & 32 & 0.0327 & 18.2 & 0.0131 & 27 & 0.0980 & 22 & 0.1277 & 36,40 & 0.0213 & 11,14 & 0.1307 \\
\hline 25 & 0.0065 & 33 & 0.0719 & 19 & 0.0261 & 28 & 0.4052 & 23 & 0.0851 & \begin{tabular}{|l|}
$37,38,39$ \\
\end{tabular} & 0.4043 & 11,15 & 0.0196 \\
\hline $\mathrm{AC}$ & 8 & 34 & 0.0327 & 20 & 0.0131 & 29 & 0.0719 & 24 & 0.0213 & $37,38,40$ & 0.0213 & 11,16 & 0.0065 \\
\hline \multirow[t]{17}{*}{ GD } & 0.6980 & 27,39 & 0.0065 & $\mathrm{AC}$ & 9 & $\mathrm{AC}$ & 9 & $\mathrm{AC}$ & 9 & 37,38 & 0.0638 & 11,17 & 0.0131 \\
\hline & & $\mathrm{AC}$ & 10 & GD & 0.6826 & GD & 0.7821 & GD & 0.7919 & 37,39 & 0.1064 & 12,13 & 0.0654 \\
\hline & & GD & 0.6537 & & & & & & & 37,40 & 0.0638 & 12,14 & 0.4183 \\
\hline & & & & & & & & & & 38,39 & 0.0213 & 12,15 & 0.1046 \\
\hline & & & & & & & & & & 38,40 & 0.0213 & 12,16 & 0.0261 \\
\hline & & & & & & & & & & 39,39 & 0.0851 & 12,18 & 0.0131 \\
\hline & & & & & & & & & & 39,40 & 0.0213 & 12,19 & 0.0065 \\
\hline & & & & & & & & & & GD & 0.813 & 13,14 & 0.0654 \\
\hline & & & & & & & & & & & & 13,15 & 0.0065 \\
\hline & & & & & & & & & & & & 13,16 & 0.0196 \\
\hline & & & & & & & & & & & & 13,17 & 0.0131 \\
\hline & & & & & & & & & & & & 13,18 & 0.0065 \\
\hline & & & & & & & & & & & & 13,19 & 0.0065 \\
\hline & & & & & & & & & & & & 13,20 & 0.0065 \\
\hline & & & & & & & & & & & & 14,16 & 0.0196 \\
\hline & & & & & & & & & & & & 14,15 & 0.0065 \\
\hline & & & & & & & & & & & & GD & 0.9600 \\
\hline
\end{tabular}

Table 2:

Allelic frequencies of Baluchi population of Pakistan based on 29 Y STRs

\begin{tabular}{|c|c|c|c|c|c|c|c|c|c|c|c|c|c|}
\hline Allele & DYS19 & Allele & DYS549 & Allele & DYS643 & Allele & DYS389I & Allele & DYS391 & Allele & DYS437 & Allele & DYS438 \\
\hline 14 & 0.2667 & 11 & 0.2000 & 10 & 0.6667 & 12 & 0.3333 & 10 & 0.7333 & 14 & 0.2667 & 9 & 0.2667 \\
\hline 15 & 0.5333 & 12 & 0.3333 & 11 & 0.2000 & 13 & 0.6000 & 11 & 0.2000 & 15 & 0.4667 & 10 & 0.4000 \\
\hline 16 & 0.2000 & 13 & 0.4667 & 12 & 0.1333 & 14 & 0.0667 & 12 & 0.0667 & 16 & 0.2667 & 11 & 0.3333 \\
\hline GD & 0.6476 & GD & 0.6762 & GD & 0.5333 & GD & 0.5619 & GD & 0.4476 & GD & 0.6857 & GD & 0.7048 \\
\hline Allele & $\begin{array}{l}\text { DYS635 } \\
\end{array}$ & Allele & GATA_H4 & Allele & DYS533 & Allele & $\begin{array}{l}\text { DYS460 } \\
\end{array}$ & Allele & DYS385b & Allele & $\begin{array}{l}\text { DYS390 } \\
\end{array}$ & Allele & DYS392 \\
\hline 21 & 0.5333 & 11 & 0.6000 & 11 & 0.3333 & 10 & 0.3333 & 14 & 0.2000 & 22 & 0.3333 & 11 & 0.5333 \\
\hline 23 & 0.2000 & 12 & 0.2667 & 12 & 0.6000 & 11 & 0.6000 & 15 & 0.1333 & 23 & 0.1333 & 12 & 0.0667 \\
\hline 24 & 0.2667 & 13 & 0.1333 & 13 & 0.0667 & 12 & 0.0667 & 16 & 0.2667 & 24 & 0.4000 & 13 & 0.0667 \\
\hline \multirow[t]{2}{*}{ GD } & 0.6476 & GD & 0.5905 & GD & 0.5619 & GD & 0.5619 & 18 & 0.4000 & 25 & 0.1333 & 14 & 0.3333 \\
\hline & & & & & & & & GD & 0.7619 & GD & 0.7429 & GD & 0.6381 \\
\hline Allele & $\begin{array}{l}\text { DYS393 } \\
\end{array}$ & Allele & DYS439 & Allele & DYS456 & Allele & DYS458 & Allele & $\begin{array}{l}\text { DYS481 } \\
\end{array}$ & Allele & $\begin{array}{l}\text { DYS448 } \\
\end{array}$ & Allele & DYS518 \\
\hline 11 & 0.2000 & 10 & 0.6000 & 13 & 0.2667 & 15 & 0.3333 & 21 & 0.0667 & 18 & 0.1333 & 36 & 0.1333 \\
\hline 12 & 0.3333 & 11 & 0.1333 & 14 & 0.2000 & 16 & 0.1333 & 22 & 0.1333 & 19 & 0.4000 & 37 & 0.1333 \\
\hline 13 & 0.2667 & 12 & 0.1333 & 15 & 0.2667 & 17 & 0.2000 & 23 & 0.6000 & 20 & 0.1333 & 38 & 0.4000 \\
\hline 14 & 0.2000 & 13 & 0.1333 & 16 & 0.2667 & 18 & 0.3333 & 24 & 0.2000 & 21 & 0.2000 & 41 & 0.2667 \\
\hline \multirow[t]{2}{*}{ GD } & 0.7905 & GD & 0.6286 & GD & 0.8000 & GD & 0.7714 & GD & 0.6191 & 22 & 0.1333 & 42 & 0.0667 \\
\hline & & & & & & & & & & GD & 0.8000 & GD & 0.7810 \\
\hline
\end{tabular}




\begin{tabular}{|c|c|c|c|c|c|c|c|c|c|c|c|c|c|}
\hline Allele & DYS627 & Allele & DYF387S1a & Allele & DYF387S1b & Allele & DYS576 & Allele & $\begin{array}{l}\text { DYS449 } \\
\end{array}$ & Allele & DYS385a & Allele & DYS389II \\
\hline 17 & 0.2000 & 35 & 0.1333 & 37 & 0.2667 & 14 & 0.1333 & 27 & 0.0667 & 9 & 0.2000 & 27 & 0.0667 \\
\hline 18 & 0.2000 & 36 & 0.5333 & 38 & 0.3333 & 15 & 0.4000 & 28 & 0.0667 & 10 & 0.0667 & 28 & 0.2667 \\
\hline 19 & 0.0667 & 37 & 0.2000 & 39 & 0.0667 & 16 & 0.0667 & 29 & 0.2000 & 11 & 0.2000 & 29 & 0.3333 \\
\hline 20 & 0.4000 & 38 & 0.0667 & 40 & 0.2667 & 17 & 0.1333 & 30 & 0.2000 & 12 & 0.0667 & 30 & 0.1333 \\
\hline 22 & 0.1333 & 39 & 0.0667 & 41 & 0.0667 & 18 & 0.1333 & 31 & 0.4000 & 13 & 0.4000 & 31 & 0.0667 \\
\hline GD & 0.7905 & GD & 0.6952 & GD & 0.7905 & 19 & 0.1333 & 33 & 0.0667 & 15 & 0.0667 & 39 & 0.1333 \\
\hline & & & & & & GD & 0.8191 & GD & 0.8000 & GD & 0.8000 & GD & 0.8286 \\
\hline
\end{tabular}

Allele 1 DYS570

\begin{tabular}{|l|l|}
\hline 13 & 0.1333 \\
\hline 14 & 0.1333 \\
\hline 15 & 0.2000 \\
\hline 16 & 0.1333 \\
\hline 17 & 0.0667 \\
\hline 18 & 0.1333 \\
\hline 19 & 0.0667 \\
\hline 20 & 0.1333 \\
\hline GD & 0.9238 \\
\hline
\end{tabular}

$\mathrm{GD}=$ Genetic diversity

Table 3:

Allelic frequencies of Mongol population of Inner Mongolia, China based on 29 Y STRs

\begin{tabular}{|c|c|c|c|c|c|c|c|c|c|c|c|c|c|}
\hline Allele & DYS391 & Allele & DYS393 & Allele & DYS460 & Allele & DYS456 & Allele & DYS437 & Allele & $\begin{array}{l}\text { DYS438 } \\
\end{array}$ & Allele & DYS19 \\
\hline 9 & 0.0667 & 12 & 0.3000 & 9 & 0.0667 & 14 & 0.3000 & 13 & 0.2000 & 9 & 0.0667 & 14 & 0.1667 \\
\hline 10 & 0.8000 & 13 & 0.3333 & 10 & 0.6667 & 15 & 0.5667 & 14 & 0.6667 & 10 & 0.8000 & 15 & 0.4333 \\
\hline 11 & 0.1333 & 14 & 0.3667 & 11 & 0.2667 & 16 & 0.1333 & 15 & 0.1333 & 11 & 0.1333 & 16 & 0.2667 \\
\hline GD & 0.3494 & GD & 0.6874 & GD & 0.4966 & GD & 0.5908 & GD & 0.5149 & GD & 0.3494 & 17 & 0.1333 \\
\hline & & & & & & & & & & & & GD & 0.7195 \\
\hline Allele & DYS385a & Allele & DYS389I & Allele & DYS390 & Allele & DYS439 & Allele & GATA_H4 & Allele & DYS549 & Allele & DYS643 \\
\hline 11 & 0.2667 & 11 & 0.0333 & 22 & 0.0333 & 10 & 0.2667 & 10 & 0.1000 & 11 & 0.0667 & 9 & 0.1333 \\
\hline 12 & 0.4000 & 12 & 0.4000 & 23 & 0.3667 & 11 & 0.4667 & 11 & 0.4000 & 12 & 0.6333 & 10 & 0.4667 \\
\hline 13 & 0.2000 & 13 & 0.4000 & 24 & 0.3000 & 12 & 0.1667 & 12 & 0.4667 & 13 & 0.2667 & 11 & 0.2667 \\
\hline 14 & 0.1333 & 14 & 0.1667 & 25 & 0.3000 & 13 & 0.1000 & 13 & 0.0333 & 14 & 0.0333 & 12 & 0.1333 \\
\hline GD & 0.7356 & GD & 0.6736 & GD & 0.7081 & GD & 0.6966 & GD & 0.6322 & GD & 0.5402 & GD & 0.6989 \\
\hline Allele & DYS635 & Allele & DYS533 & Allele & DYS389II & Allele & DYS392 & Allele & $\begin{array}{l}\text { DYS448 } \\
\end{array}$ & Allele & $\begin{array}{l}\text { DYS458 } \\
\end{array}$ & Allele & DYS576 \\
\hline 19 & 0.0333 & 9 & 0.0667 & 27 & 0.0667 & 7 & 0.0333 & 17 & 0.0333 & 14 & 0.0333 & 15 & 0.2000 \\
\hline 20 & 0.0667 & 10 & 0.0667 & 28 & 0.2667 & 11 & 0.2000 & 18 & 0.1667 & 15 & 0.0667 & 16 & 0.1333 \\
\hline 21 & 0.2667 & 11 & 0.5000 & 29 & 0.5000 & 12 & 0.1667 & 19 & 0.2667 & 16 & 0.2667 & 17 & 0.3333 \\
\hline 22 & 0.3333 & 12 & 0.3000 & 30 & 0.0667 & 13 & 0.4333 & 20 & 0.2667 & 17 & 0.2333 & 18 & 0.2333 \\
\hline
\end{tabular}




\begin{tabular}{|c|c|c|c|c|c|c|c|c|c|c|c|c|c|}
\hline 23 & 0.3000 & 13 & 0.0667 & 31 & 0.0333 & 14 & 0.1333 & 21 & 0.1000 & 18 & 0.3333 & 19 & 0.0667 \\
\hline GD & 0.7471 & GD & 0.6690 & 32 & 0.0667 & 16 & 0.0333 & 22 & 0.1667 & 19 & 0.0667 & 20 & 0.0333 \\
\hline & & & & GD & 0.6874 & GD & 0.7494 & GD & 0.8184 & GD & 0.7793 & GD & 0.7977 \\
\hline Allele & DYS481 & Allele & DYS570 & Allele & DYF387s1b & Allele & DYS627 & Allele & DYF387s1a & Allele & DYS385b & Allele & DYS449 \\
\hline 20 & 0.0667 & 15 & 0.0333 & 36 & 0.0333 & 19 & 0.1667 & 34 & 0.0333 & 11 & 0.0667 & 26 & 0.0667 \\
\hline 21 & 0.1000 & 16 & 0.2000 & 37 & 0.1333 & 20 & 0.1333 & 35 & 0.1000 & 12 & 0.2000 & 27 & 0.3333 \\
\hline 22 & 0.1000 & 17 & 0.1000 & 38 & 0.1667 & 21 & 0.2333 & 36 & 0.3333 & 13 & 0.3333 & 28 & 0.1333 \\
\hline 23 & 0.1333 & 18 & 0.1333 & 39 & 0.3000 & 22 & 0.3000 & 37 & 0.3000 & 14 & 0.0333 & 29 & 0.1667 \\
\hline 24 & 0.1667 & 19 & 0.4000 & 40 & 0.2333 & 23 & 0.1000 & 38 & 0.0667 & 16 & 0.0333 & 30 & 0.0667 \\
\hline 25 & 0.4333 & 20 & 0.1333 & 41 & 0.1333 & 24 & 0.0333 & 39 & 0.1000 & 17 & 0.1667 & 31 & 0.0667 \\
\hline GD & 0.7678 & GD & 0.7793 & GD & 0.6989 & 25 & 0.0333 & 41 & 0.0667 & 18 & 0.0333 & 32 & 0.0333 \\
\hline & & & & & & GD & 0.8253 & GD & 0.5402 & 20 & 0.0333 & 33 & 0.0667 \\
\hline & & & & & & & & & & 21 & 0.1000 & 34 & 0.0333 \\
\hline & & & & & & & & & & GD & 0.8299 & 35 & 0.0333 \\
\hline & & & & & & & & & & & & GD & 0.8506 \\
\hline Allele & DYS518 & & & & & & & & & & & & \\
\hline 33 & 0.0333 & & & & & & & & & & & & \\
\hline 35 & 0.0667 & & & & & & & & & & & & \\
\hline 36 & 0.1000 & & & & & & & & & & & & \\
\hline 37 & 0.2000 & & & & & & & & & & & & \\
\hline 38 & 0.2667 & & & & & & & & & & & & \\
\hline 39 & 0.1667 & & & & & & & & & & & & \\
\hline 40 & 0.0333 & & & & & & & & & & & & \\
\hline 41 & 0.0333 & & & & & & & & & & & & \\
\hline 42 & 0.0333 & & & & & & & & & & & & \\
\hline 43 & 0.0333 & & & & & & & & & & & & \\
\hline 44 & 0.0333 & & & & & & & & & & & & \\
\hline GD & 0.8690 & & & & & & & & & & & & \\
\hline
\end{tabular}

Table 4:

Forensic statistical parameters in Hazara population of Pakistan on 8 levels

\begin{tabular}{|c|c|c|c|c|c|c|c|c|}
\hline Haplotypes & MH 9 Loci & $\begin{array}{l}\text { SWGDAM } \\
11 \text { loci }\end{array}$ & $\begin{array}{l}\text { PowerPlex } \\
\text { Y12 loci }\end{array}$ & $Y$-filer 17 loci & $\begin{array}{l}\text { PowerPlex } \\
\text { Y23 loci }\end{array}$ & 26-Y Loci & $\begin{array}{l}\text { Y-filer Plus } \\
27 \text { loci }\end{array}$ & 30 Y Loci \\
\hline Sample size & 153 & 153 & 153 & 153 & 153 & 153 & 47 & 47 \\
\hline $\begin{array}{l}\text { Haplotype } \\
\text { diversity }\end{array}$ & $\begin{array}{l}0.9316+/- \\
0.0157\end{array}$ & $\begin{array}{l}0.9485+/- \\
0.0129\end{array}$ & $\begin{array}{l}0.9485+/- \\
0.0129\end{array}$ & $\begin{array}{l}0.9942+/- \\
0.0019\end{array}$ & $\begin{array}{l}0.9981+/- \\
0.0012\end{array}$ & $\begin{array}{l}0.9985+/- \\
0.0012\end{array}$ & $\begin{array}{l}0.9991+/- \\
0.0046\end{array}$ & $\begin{array}{l}1.0000+/- \\
0.0044\end{array}$ \\
\hline$R M P$ & 0.07442128 & 0.0577134 & 0.0577134 & 0.01231458 & 0.00835716 & 0.00800872 & 0.02223081 & 0.0213234 \\
\hline$D C$ & 0.470588235 & 0.529411765 & 0.529411765 & 0.764705882 & 0.91503268 & 0.941176471 & 0.978723404 & 1 \\
\hline Haplotypes & 72 & 81 & 81 & 117 & 140 & 144 & 46 & 47 \\
\hline $\begin{array}{l}\text { Unique } \\
\text { Haplotype }\end{array}$ & 54 & 63 & 64 & 97 & 132 & 140 & 45 & 47 \\
\hline $\begin{array}{l}\text { \% of Unique } \\
\text { Haplotype }\end{array}$ & 0.75 & 0.777777778 & 0.790123457 & 0.829059829 & 0.942857143 & 0.972222222 & 0.97826087 & 100 \\
\hline
\end{tabular}

Table 5: 
Forensic statistical parameters in Balochi population of Pakistan on 8 levels

\begin{tabular}{|l|l|l|l|l|l|l|l|l|}
\hline & MH 9 Loci & $\begin{array}{l}\text { SWGDAM } \\
\text { II loci }\end{array}$ & $\begin{array}{l}\text { PowerPlex } \\
\text { Y12 loci }\end{array}$ & Y-filer 17 loci & $\begin{array}{l}\text { PowerPlex } \\
\text { Y23 loci }\end{array}$ & 26-Y Loci & $\begin{array}{l}\text { Y-filer Plus } \\
\text { 27 loci }\end{array}$ & 29-Y Loci \\
\hline Sample size & 15 & 15 & 15 & 15 & 15 & 15 & 15 & 15 \\
\hline $\begin{array}{l}\text { Gene } \\
\text { diversity }\end{array}$ & $0.9810+/-$ & $1.0000+/-$ & $1.0000+/-$ & $\begin{array}{l}1.0000+/- \\
0.0243\end{array}$ & $\begin{array}{l}1.0000+/- \\
0.0243\end{array}$ & $\begin{array}{l}1.0000+/- \\
0.0243\end{array}$ & $\begin{array}{l}1.0000+/- \\
0.0243\end{array}$ & $\begin{array}{l}1.0000+/- \\
0.0243\end{array}$ \\
\hline RMP & 0.0844 & 0.0667 & 0.0667 & 0.0667 & 0.0667 & 0.0667 & 0.0667 & 0.0667 \\
\hline DC & 0.866 & 1 & 1 & 1 & 1 & 1 & 1 & 1 \\
\hline Haplotypes & 13 & 15 & 15 & 15 & 15 & 15 & 15 & 15 \\
\hline $\begin{array}{l}\text { Unique } \\
\text { Haplotype }\end{array}$ & 11 & 15 & 15 & 15 & 15 & 15 & 15 & 15 \\
\hline $\begin{array}{l}\text { \% of Unique } \\
\text { Haplotype }\end{array}$ & $0.85 \%$ & $100 \%$ & $100 \%$ & $100 \%$ & $100 \%$ & $100 \%$ & $100 \%$ & $100 \%$ \\
\hline
\end{tabular}

\section{Table 6:}

Forensic statistical parameters in Mongol population of Inner Mongolia China on 8 levels

\begin{tabular}{|c|c|c|c|c|c|c|c|c|}
\hline & MH 9 Loci & $\begin{array}{l}\text { SWGDAM } \\
11 \text { loci }\end{array}$ & $\begin{array}{l}\text { PowerPlex } \\
\text { Y12 loci }\end{array}$ & Y-filer 17 loci & $\begin{array}{l}\text { PowerPlex } \\
\text { Y23 loci }\end{array}$ & $26-Y$ Loci & $\begin{array}{l}\text { Y-filer Plus } \\
27 \text { loci }\end{array}$ & 29-Y Loci \\
\hline Sample size & 30 & 30 & 30 & 30 & 30 & 30 & 30 & 30 \\
\hline $\begin{array}{l}\text { Gene } \\
\text { diversity }\end{array}$ & $\begin{array}{l}0.9931+/- \\
0.0105\end{array}$ & $\begin{array}{l}0.9931+/- \\
0.0105\end{array}$ & $\begin{array}{l}0.9931+/- \\
0.0105\end{array}$ & $\begin{array}{l}0.9977+/- \\
0.0094\end{array}$ & $\begin{array}{l}1.0000+/- \\
0.0086\end{array}$ & $\begin{array}{l}1.0000+/- \\
0.0086\end{array}$ & $\begin{array}{l}1.0000+/- \\
0.0086\end{array}$ & $\begin{array}{l}1.0000+/- \\
0.0086\end{array}$ \\
\hline$R M P$ & 0.04 & 0.04 & 0.04 & 0.0356 & 0.0332667 & 0.0332667 & 0.0332667 & 0.0332667 \\
\hline$D C$ & 0.9 & 0.9 & 0.9 & 0.966666667 & 1 & 1 & 1 & 1 \\
\hline Haplotypes & 27 & 27 & 27 & 29 & 30 & 30 & 30 & 30 \\
\hline $\begin{array}{l}\text { Unique } \\
\text { Haplotype }\end{array}$ & 24 & 24 & 24 & 28 & 30 & 30 & 30 & 30 \\
\hline $\begin{array}{l}\text { \% of Unique } \\
\text { Haplotype }\end{array}$ & 0.8888888889 & 0.8888888889 & 0.888888889 & 0.965517241 & 100 & 100 & 100 & 100 \\
\hline
\end{tabular}




\section{Table 7:}

Pairwise Rst values between Hazara Population of Pakistan and other 27 reference populations

\begin{tabular}{|c|c|c|c|c|c|c|c|c|c|c|c|c|c|c|c|c|c|c|c|c|c|c|c|c|c|c|c|c|}
\hline & {$[1]$} & {$[2]$} & [3] & {$[4]$} & {$[5]$} & [6] & {$[7]$} & {$[8]$} & [9] & {$[10]$} & [11] & {$[12]$} & {$[13]$} & [14] & [15] & [16] & {$[17]$} & [18] & [19] & [20] & [21] & {$[22]$} & {$[23]$} & {$[24]$} & {$[25]$} & {$[26]$} & {$[27]$} & {$[28]$} \\
\hline [1] & & $\frac{1-1}{-1}$ & -1 & $\frac{1-1}{-1}$ & $\frac{1}{-1}$ & -1 & -1 & $\frac{1}{-1}$ & -1 & -1 & -1 & -1 & -1 & -1 & -1 & -1 & -1 & -1 & -1 & -1 & -1 & -1 & -1 & -1 & -1 & -1 & -1 & -1 \\
\hline$[2]$ & 0.013 & & -1 & -1 & -1 & -1 & -1 & -1 & -1 & -1 & -1 & -1 & -1 & -1 & -1 & -1 & -1 & -1 & -1 & -1 & -1 & -1 & -1 & -1 & -1 & -1 & -1 & -1 \\
\hline [3] & 0.026 & 0.058 & & -1 & -1 & -1 & -1 & -1 & -1 & -1 & -1 & -1 & -1 & -1 & -1 & -1 & -1 & -1 & -1 & -1 & -1 & -1 & -1 & -1 & -1 & -1 & -1 & -1 \\
\hline$[4]$ & 0.113 & 0.149 & 0.140 & & -1 & -1 & -1 & -1 & -1 & -1 & -1 & -1 & -1 & -1 & -1 & -1 & -1 & -1 & -1 & -1 & -1 & -1 & -1 & -1 & -1 & -1 & -1 & -1 \\
\hline$[5]$ & 0.094 & 0.147 & 0.143 & 0.006 & & -1 & -1 & -1 & -1 & -1 & -1 & -1 & -1 & -1 & -1 & -1 & -1 & -1 & -1 & -1 & -1 & -1 & -1 & -1 & -1 & -1 & -1 & -1 \\
\hline [6] & 0.147 & 0.152 & 0.142 & 0.010 & 0.004 & & -1 & -1 & -1 & -1 & -1 & -1 & -1 & -1 & -1 & -1 & -1 & -1 & -1 & -1 & -1 & -1 & -1 & -1 & -1 & -1 & -1 & -1 \\
\hline [7] & 0.030 & 0.113 & 0.161 & 0.056 & 0.057 & 0.066 & & -1 & -1 & -1 & -1 & -1 & -1 & -1 & -1 & -1 & -1 & -1 & -1 & -1 & -1 & -1 & -1 & -1 & -1 & -1 & -1 & -1 \\
\hline$[8]$ & 0.038 & 0.038 & 0.073 & 0.068 & 0.059 & 0.072 & 0.054 & & -1 & -1 & -1 & -1 & -1 & -1 & -1 & -1 & -1 & -1 & -1 & -1 & -1 & -1 & -1 & -1 & -1 & -1 & -1 & -1 \\
\hline$\left[\begin{array}{l}{[8]} \\
{[9]}\end{array}\right.$ & $\begin{array}{l}0.038 \\
0.036\end{array}$ & $\begin{array}{l}0.038 \\
0.095\end{array}$ & 0.0010 & $\begin{array}{l}0.068 \\
0.012\end{array}$ & 0.0596 & 0.0125 & $\begin{array}{l}0.044 \\
0.047\end{array}$ & 0.027 & & $\frac{-1}{-1}$ & $\frac{-1}{-1}$ & $\frac{-1}{-1}$ & $\frac{-1}{-1}$ & $\frac{-1}{-1}$ & $\frac{-1}{-1}$ & $\frac{-1}{-1}$ & $\frac{-1}{-1}$ & $\frac{-1}{-1}$ & $\frac{-1}{-1}$ & $\frac{-1}{-1}$ & $\frac{-1}{-1}$ & $\frac{-1}{-1}$ & $\frac{-1}{-1}$ & $\frac{-1}{-1}$ & $\frac{-1}{-1}$ & $\frac{-1}{-1}$ & $\frac{-1}{-1}$ & $\begin{array}{l}-1 \\
-1\end{array}$ \\
\hline [10] & 0.023 & 0.033 & 0.057 & 0.048 & 0.042 & 0.053 & 0.042 & 0.006 & 0.013 & & -1 & -1 & -1 & -1 & -1 & -1 & -1 & -1 & -1 & -1 & -1 & -1 & -1 & -1 & -1 & -1 & -1 & -1 \\
\hline$[11]$ & 0.051 & 0.023 & $\begin{array}{l}0.0071 \\
0.046\end{array}$ & $\frac{0.040}{0.094}$ & 0.093 & 0.098 & 0.080 & 0.032 & 0.050 & 0.017 & & -1 & -1 & -1 & -1 & -1 & -1 & -1 & -1 & -1 & -1 & -1 & -1 & -1 & -1 & -1 & -1 & -1 \\
\hline$[12]$ & 0.058 & 0.290 & 0.288 & 0.071 & 0.090 & 0.082 & 0.1110 & 0.183 & 0.112 & 0.142 & 0.187 & & -1 & -1 & -1 & -1 & -1 & -1 & -1 & -1 & -1 & -1 & -1 & -1 & -1 & -1 & -1 & -1 \\
\hline [13] & 0.005 & 0.000 & 0.072 & 0.127 & 0.126 & 0.131 & 0.083 & 0.029 & 0.071 & 0.019 & 0.027 & 0.270 & & -1 & -1 & -1 & -1 & -1 & -1 & -1 & -1 & -1 & -1 & -1 & -1 & -1 & -1 & -1 \\
\hline$[14]$ & 0.014 & 0.079 & 0.177 & 0.177 & 0.193 & 0.191 & $\begin{array}{l}0.005 \\
0.093\end{array}$ & 0.025 & 0.131 & 0.083 & 0.089 & 0.200 & 0.065 & & -1 & -1 & -1 & -1 & -1 & -1 & $\frac{-1}{-1}$ & $\frac{-1}{-1}$ & $\frac{-1}{-1}$ & $\frac{-1}{-1}$ & $\frac{-1}{-1}$ & $\frac{-1}{-1}$ & $\frac{-1}{-1}$ & $\frac{-1}{-1}$ \\
\hline [15] & 0.016 & 0.028 & 0.098 & 0.191 & 0.187 & 0.194 & 0.145 & 0.045 & 0.123 & 0.064 & 0.069 & 0.342 & 0.040 & 0.084 & & -1 & -1 & -1 & -1 & -1 & -1 & -1 & -1 & -1 & -1 & -1 & -1 & -1 \\
\hline$\left[\begin{array}{lll}{[16]} \\
{[16]}\end{array}\right.$ & 0.016 & 0.203 & 0.164 & 0.126 & 0.153 & 0.136 & 0.242 & 0.161 & 0.110 & 0.1114 & 0.122 & 0.272 & 0.194 & 0.319 & 0.263 & & -1 & -1 & -1 & -1 & -1 & -1 & -1 & -1 & -1 & -1 & -1 & -1 \\
\hline$[17]$ & 0.004 & 0.004 & 0.062 & 0.156 & 0.158 & 0.161 & 0.126 & 0.026 & 0.089 & 0.030 & 0.017 & 0.325 & 0.005 & 0.083 & 0.009 & 0.184 & . & -1 & -1 & -1 & -1 & -1 & -1 & -1 & -1 & -1 & -1 & -1 \\
\hline$[18]$ & 0.045 & 0.095 & 0.101 & 0.161 & 0.181 & 0.172 & 0.181 & 0.115 & 0.112 & 0.087 & 0.043 & 0.259 & 0.097 & 0.169 & 0.161 & 0.109 & 0.084 & & -1 & -1 & -1 & -1 & -1 & -1 & -1 & -1 & -1 & -1 \\
\hline [19] & 0.005 & 0.054 & 0.106 & 0.092 & 0.091 & 0.088 & 0.111 & 0.045 & 0.051 & 0.024 & 0.018 & 0.255 & 0.050 & 0.156 & 0.112 & 0.114 & 0.054 & 0.038 & & -1 & -1 & -1 & -1 & -1 & -1 & -1 & -1 & -1 \\
\hline$\left[\begin{array}{l}[1]] \\
{[20]}\end{array}\right.$ & 0.0457 & $\begin{array}{l}0.0534 \\
0.054\end{array}$ & 0.059 & 0.094 & 0.1961 & 0.1806 & 0.111 & $\begin{array}{l}0.0453 \\
0.092\end{array}$ & $\begin{array}{l}0.051 \\
0.129\end{array}$ & 0.024 & 0.028 & 0.325 & 0.085 & 0.1508 & $\frac{0.112}{0.101}$ & 0.114 & $\begin{array}{l}0.054 \\
0.039\end{array}$ & $\frac{0.050}{0.040}$ & 0.078 & & -1 & -1 & -1 & -1 & -1 & -1 & -1 & -1 \\
\hline$[21]$ & 0.060 & 0.070 & 0.089 & 0.138 & 0.153 & 0.148 & 0.150 & 0.086 & 0.094 & 0.065 & 0.022 & 0.242 & 0.078 & 0.139 & 0.131 & 0.127 & 0.056 & 0.006 & 0.032 & 0.026 & & -1 & -1 & -1 & -1 & -1 & -1 & -1 \\
\hline$[22]$ & 0.005 & 0.139 & 0.134 & 0.306 & 0.319 & 0.302 & 0.326 & 0.183 & 0.232 & 0.166 & 0.1119 & 0.536 & 0.1993 & 0.274 & 0.148 & 0.371 & 0.130 & 0.129 & 0.271 & 0.085 & 0.139 & & -1 & -1 & - & -1 & -1 & -1 \\
\hline$\left[\begin{array}{lll}{[23]} \\
{[23]}\end{array}\right.$ & 0.027 & $\begin{array}{l}0.1353 \\
0.263\end{array}$ & 0.3546 & $\frac{0.300}{0.302}$ & $\begin{array}{l}.3158 \\
.338\end{array}$ & 0.302 & 0.3558 & $\begin{array}{l}0.105 \\
0.235\end{array}$ & 0.2526 & 0.100 & 0.11203 & $\frac{0.350}{0.550}$ & $\begin{array}{l}.15287 \\
\end{array}$ & 0.3742 & $\begin{array}{l}0.140 \\
0.282\end{array}$ & 0.351 & 0.1544 & 0.239 & 0.289 & $\begin{array}{l}0.207 \\
0.207\end{array}$ & 0.159 & 0.668 & & -1 & -1 & -1 & -1 & -1 \\
\hline [24] & 0.003 & 0.107 & 0.108 & 0.267 & 0.279 & 0.260 & 0.283 & 0.155 & 0.199 & 0.135 & 0.084 & 0.490 & 0.149 & 0.230 & 0.135 & 0.310 & 0.098 & 0.091 & 0.206 & 0.061 & 0.099 & 0.008 & 0.630 & & -1 & -1 & -1 & -1 \\
\hline$[25]$ & 0.010 & 0.037 & 0.069 & 0.210 & 0.205 & 0.200 & 0.210 & 0.072 & 0.141 & 0.076 & 0.052 & 0.422 & 0.068 & $\begin{array}{l}0.250 \\
0.185\end{array}$ & 0.135 & 0.285 & 0.0980 & $\begin{array}{l}0.091 \\
0.122\end{array}$ & $\begin{array}{l}.206 \\
0.118\end{array}$ & 0.001 & 0.099 & 0.008 & $\begin{array}{l}0.030 \\
0.379\end{array}$ & 0.083 & & $\frac{-1}{-1}$ & $\frac{-1}{-1}$ & $\frac{-1}{-1}$ \\
\hline$[26]$ & 0.010 & 0.053 & 0.076 & 0.213 & 0.217 & 0.208 & 0.214 & 0.092 & 0.147 & 0.085 & 0.041 & 0.420 & 0.089 & 0.181 & 0.076 & 0.278 & 0.035 & 0.092 & 0.127 & 0.027 & 0.073 & 0.065 & 0.382 & 0.039 & 0.014 & & -1 & -1 \\
\hline 1רכי & 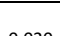 & 7 & 20 & ברכר & 0 & محכ & م & 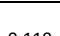 & ברבר & 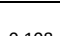 & 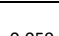 & ברבי & F & 0 & 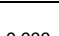 & ברב & 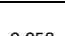 & 7 & 12 & 0 & 保 & 0 & 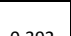 & 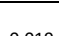 & 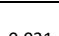 & -1 & & 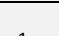 \\
\hline $\begin{array}{l}[2]] \\
{[28]}\end{array}$ & 0.020 & 0.008 & $\begin{array}{l}0.1059 \\
0.059\end{array}$ & $\begin{array}{l}.281 \\
0.196\end{array}$ & 0.419 & 0.250 & 0.140 & $\begin{array}{l}0.119 \\
0.071\end{array}$ & 0.137 & 0.072 & $\begin{array}{l}0.035 \\
0.035\end{array}$ & 0.4271 & 0.0050 & 0.192 & $\begin{array}{l}0.0999 \\
0.053\end{array}$ & 0.249 & $\begin{array}{l}0.058 \\
0.014\end{array}$ & $\frac{0.097}{0.100}$ & 0.124 & 0.0495 & 0.080 & $\begin{array}{l}0.048 \\
0.083\end{array}$ & 0 & $\begin{array}{l}0.0055 \\
0.055\end{array}$ & $\begin{array}{l}0.031 \\
0.001\end{array}$ & $\begin{array}{l}0.000 \\
\end{array}$ & 0.020 & -1 \\
\hline
\end{tabular}

[1] Hazara,Balochistan, Pakistan; [2] Afghanistan [Afghan]; [3] Afghanistan [Pathan]; [4] Beijing, China [Han];[5] China [Han]; [6]Shanghai, China [Han]; [7]

Xinjiang, China [Kazakh]; [8] Inner Mongolia, China [Mongolian]; [9]Liaoning, China [Mongolian]; [10] Ordos-Western Inner Mongolia, China [Mongolian]; [11]

Xinjiang, China [Uighur];[12] East Kazakhstan, Kazakhstan [Kazakh]; [13] Kazakhstan [Kazakh]; [14] South Kazakhstan, Kazakhstan [Kazakh];[15] Ulaanbaatar,

Mongolia [Mongolian]; [16] Swat and Dir District, Pakistan [Gujjar]; [17]Balochistan, Pakistan [Hazara]; [18] Azad Kashmir, Pakistan [Kashmiri]; [19] Swat and

Dir District, Pakistan [Kohistani]; [20] Pakistan [Pathan]; [21] Punjab, Pakistan [Punjabi]; [22]Swat and Dir District, Pakistan [Tharklani, Pashtun] ;[23] Swat and

Dir District, Pakistan [Uthmankheil, Pashtun]; [24] Swat and Dir District, Pakistan [Yousafzai, Pashtun]; [25] Archangelsk, Russian Federation [Russian]; [26]

Lipezk, Russian Federation [Russian]; [27] Pensa, Russian Federation [Russian]; [28] Russian Federation [Russian] 


\section{Table 8:}

Frequencies of null allele at DYS448 in various ethnic groups across continents

\begin{tabular}{|c|c|c|c|c|c|}
\hline Continent & Population & $\begin{array}{l}\text { Number of } \\
\text { samples }\end{array}$ & No of del & $\%$ & Reference \\
\hline \multirow[t]{10}{*}{ Asia } & Hazara & 153 & 26 & $16.59 \%$ & Current Study \\
\hline & Korean & 708 & 6 & $0.85 \%$ & $\begin{array}{l}\text { Myung Jin Park et } \\
\text { al .,2005 }\end{array}$ \\
\hline & Kalmykia & 99 & 7 & $7.07 \%$ & Roewer et al.,2007 \\
\hline & Japan & 1079 & 10 & $0.92 \%$ & Mizuno et al.,2007 \\
\hline & Malaysia & 980 & 3 & $0.30 \%$ & Chang et al.,2007 \\
\hline & Nepal & 769 & 3 & $0.39 \%$ & Parkin et al.,2007 \\
\hline & Tajikistan & 124 & 3 & $2.41 \%$ & Balaresque et al.,2008 \\
\hline & Kyrgyzstan & 87 & 9 & 10.34 & Balaresque et al.,2008 \\
\hline & China & 130 & 3 & $2.30 \%$ & Balaresque et al.,2008 \\
\hline & Asian & 330 & 2 & $0.61 \%$ & $\begin{array}{l}\text { AmpFlSTR } \AA \text { YfilerTM } \\
\text { database }\end{array}$ \\
\hline Europe & Spain & 247 & 1 & $0.40 \%$ & Sanchez et al.,2007 \\
\hline Africa & Egypt & 208 & 1 & $0.48 \%$ & Omran et al.,2008 \\
\hline \multirow[t]{4}{*}{ Americas } & Mexico & 326 & 1 & $0.30 \%$ & $\begin{array}{l}\text { Gutierrez-Alarcon et } \\
\text { al.,2007 }\end{array}$ \\
\hline & $\begin{array}{l}\text { African } \\
\text { American }\end{array}$ & 985 & 2 & $0.20 \%$ & $\begin{array}{l}\text { AmpFlSTR } \AA \text { Yfiler } \\
\text { database }\end{array}$ \\
\hline & $\begin{array}{l}\text { Caucasian } \\
\text { (USA) }\end{array}$ & 1276 & 2 & $0.16 \%$ & $\begin{array}{l}\text { AmpFlSTR }{ }^{\circledR} \text { Yfiler }^{\mathrm{TM}} \\
\text { database }\end{array}$ \\
\hline & & 7501 & 79 & $1.05 \%$ & \\
\hline
\end{tabular}


Table 9:

Sequence in the relevant flanking and repeat region of the DYS448 locus for normal and null alleles

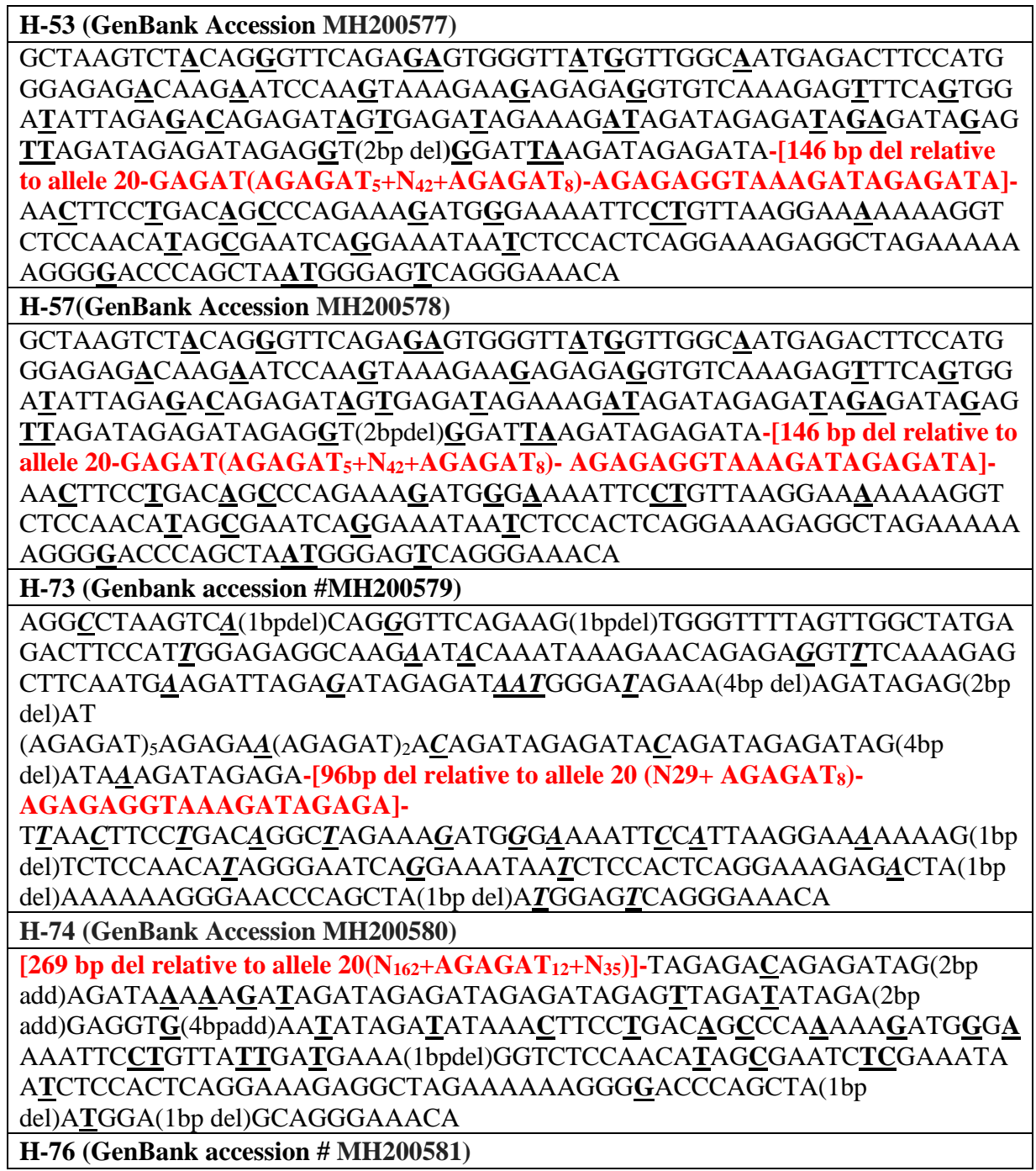


GGCCTAAGTCT $\underline{A} C A G \underline{G} G T T C A G A \underline{G A G T G G G T T} \underline{\boldsymbol{A}} \underline{\boldsymbol{G}} \underline{\mathbf{G}} \mathrm{GTTGGC} \underline{\boldsymbol{A} A T G A G A C T T C C A T}$ GGGAGAG $\underline{A} C A A G \underline{A} A T C C A A \underline{G}$ TAAAGAA $\underline{G}$ AGAGA $\underline{G}$ GTGTCAAAGAG $\underline{T}$ TTCA $\underline{G}$ TG GATAT-[157bp del relative to allele 20( $\left.\left.\mathrm{N}_{47}+\overline{A G A G A T}_{12}+\mathbf{N}_{36}\right)\right]-$

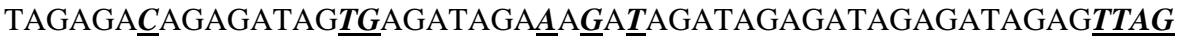

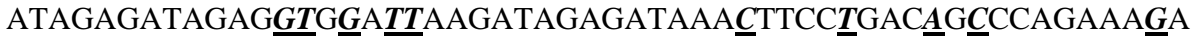

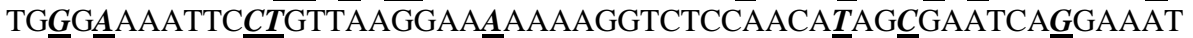
AA $\underline{\boldsymbol{T}} \mathrm{CT}$ CCACTCAGGAAAGAGGCTAGAAAAAAGGGGACCCAGCTA(1 $\boldsymbol{b p}$ del)ATGGAGTCAGGGAAACAGG

H-82 (GenBank accession \# MH200582)

GAAGGTGGGTTTTAGTTGGCTATGAGACTTCCATGGGAGAGGCAAGGATCCAAA TAAAGAACAGAGAAGTGTCAAAGAGCTTCAATGGAGATTAGAAATAGAGATCGC GAGACAGAAAGGGAGATAGAGACATGGATAA(AGAGAT) ${ }_{12}-\mathrm{N}_{42}-($ AGAGAT) 8 AGAGAGGTAAAGATAGAGATAAATTTCCAGACCGGCCAGAAATATGAGGAAATT CACGTTAAGGAAGAAAAGGTCTCCAACACAGGGAATCA

\section{H-84 (GenBank Accession MH200583)}

[272 bp del relative to allele 20( $\mathbf{N}_{162}+$ AGAGAT $\left._{12}+\mathbf{N}_{38}\right)$ ]-AGACAGAGATAG(2bp

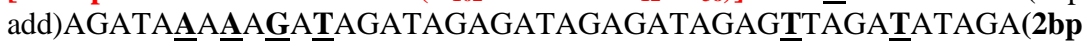
add)GAGGT鸟 $(\overline{\mathbf{b}} \mathbf{p}$

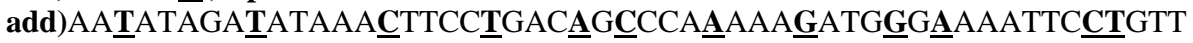

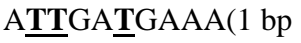

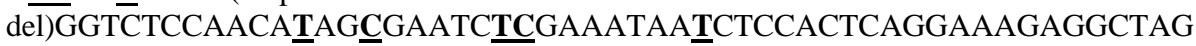
AAAAAAGGGGACCCAGCTA(1 bp del)ATGGA-GCAGGGAAACA

H-85(GenBank Accession MH200584)

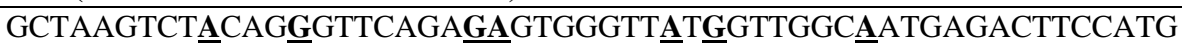

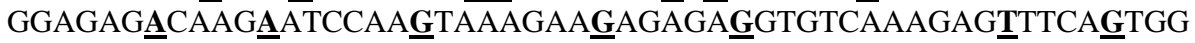

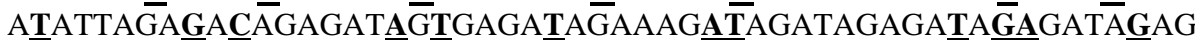

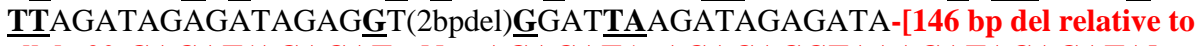
allele 20-GAGAT(AGAGAT ${ }_{5}+\mathrm{N}_{42}+\mathrm{AGAGAT}$ )- AGAGAGGTAAAGATAGAGATA]-

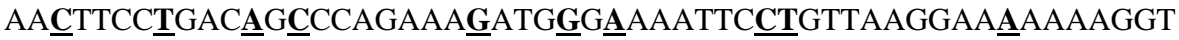

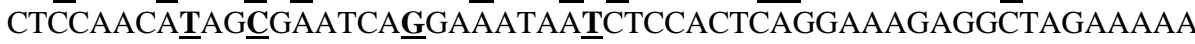
AGGGGACCCAGCTAATGGGAGTCAGGGAAACA

H-86 (Genbank accession MH200585)

GGCTAAGTCÁ(1bpdel)CAGGGTTCAGAAG(1bpdel)TGGGTTTTAGTTGGCTATGAGA

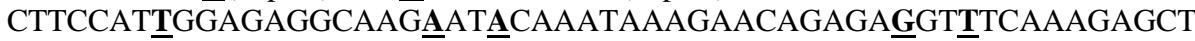

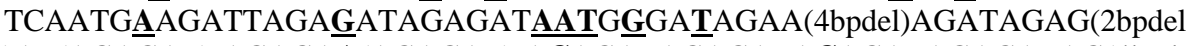
)AT(AGAGAT) ${ }_{5}$ AGAGA $\underline{\mathbf{A}}$ (AGAGAT) ${ }_{2}$ A $\underline{\text { CAGATAGAGATA }}$ AAGATAGAGATAG(4bpde 1)ATAAAGATAGAGA-[96 bp del relative to allele 20 (N29+ AGAGAT $_{8}$ )-

AGAGAGGTAAAGATAGAGA]-

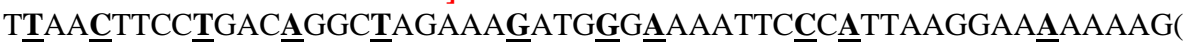

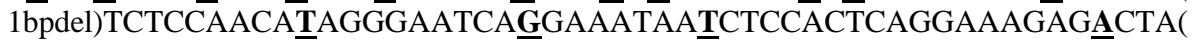
1bpdel)AAAAAAGGGAACCCAGCTĀ $\underline{\text { ATGGAG }}$ TCAGGGAAACAG

H-89 (GenBank Accession MH200586)

[269 bp del relative to allele $20\left(\mathrm{~N}_{162}+\mathrm{AGAGAT}_{12}+\mathrm{N}_{35}\right)$ ]TAGAGA $\underline{\mathbf{C}} A G A G A T A G \underline{T} G A T A \underline{T} A G A \underline{A} A G A(2 b p$

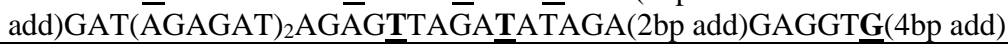




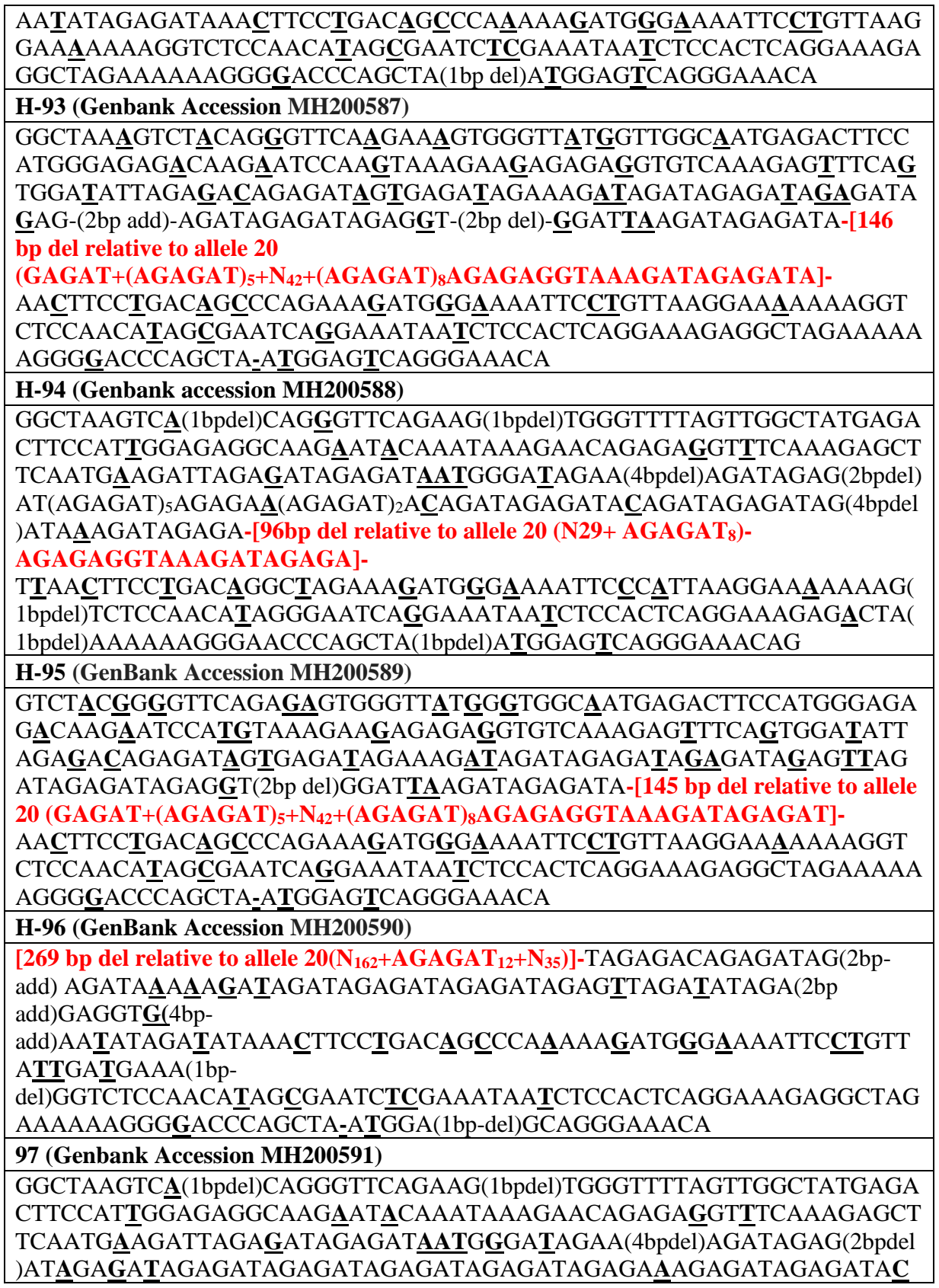




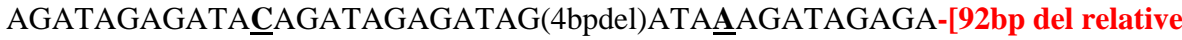
to allele 20 (N29+ AGAGAT) 8 - AGAGAGGTAAAGATA]-

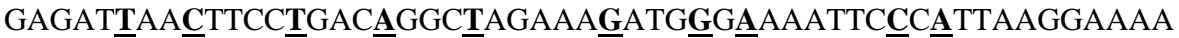

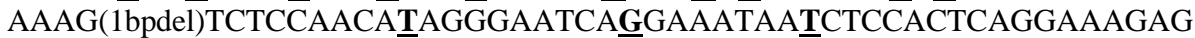
ACTA(1bpdel)AAAAAAGGGAACCCAGCTA(1bpdel)A $\underline{\text { TGGAG }}$ TCAGGGAAACAGG $\underline{\mathbf{A}}$ AG

100 (Genbank Accession MH200592)

GGCTAAGTC $\underline{A}$ (1bpdel)CAGGGTTCAGAAG(1bpdel)TGGGTTTTAGTTGGCTATGAGA

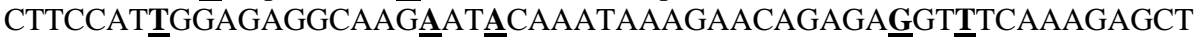
TCAATGĀA GATTAGAGATAGAGATAATGGGATAGAA(4bpdel)AGĀTAGAG(1bpdel

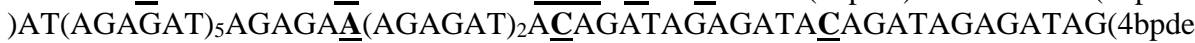
1)ATAAAGATAGAGA-[96 bp del relative to allele 20 (N29+ AGAGAT $_{8}$ -

AGAGAGgTAAAGATAGAGA]-

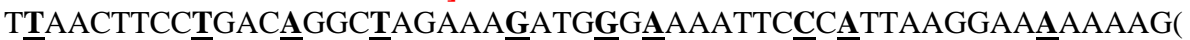

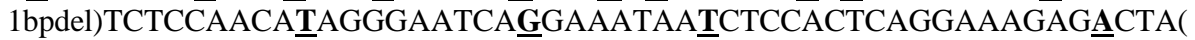
1bpdel)AAAAAAGGGAACCCAGCTĀ(1bpdel)ATGGAGTCAGGGAAACA

102 (Genbank Accession MH200593)

CTAAGTCÁ(1bpdel)CAGGGTTCAGAAG(1bpdel)TGGGTTTTAGTTGGCTATGAGACT

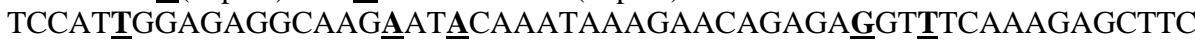

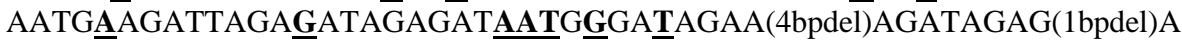

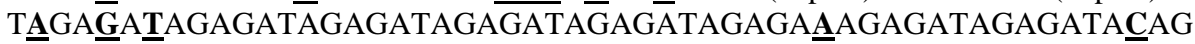

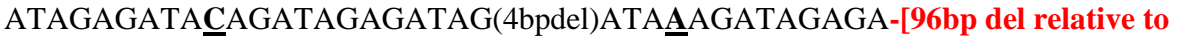
allele 20 (N29+ AGAGAT) ) AGAGAGGTAAAGATAGAGA]-

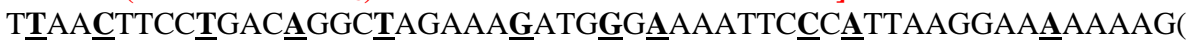

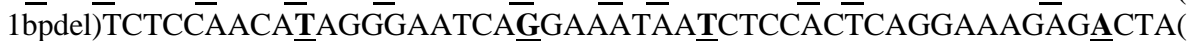
1bpdel)AAAAAAGGGAACCCAGCTA(1bpdel)A

H-103(GenBank Accession MH200594)

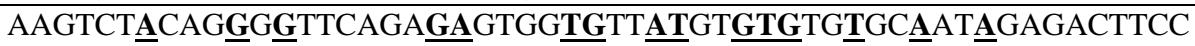

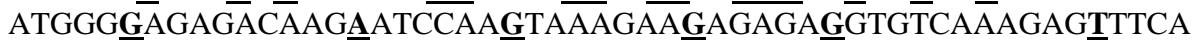

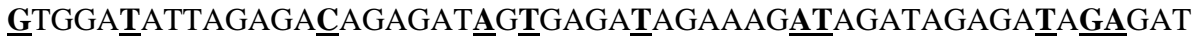

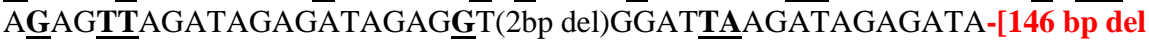
relative to allele 20

$(\text { GAGAT+(AGAGAT) })_{5}+\mathbf{N}_{42}+\left(\right.$ AGAGAT) $_{8}$ AGAGAGGTAAAGATAGAGATA]-

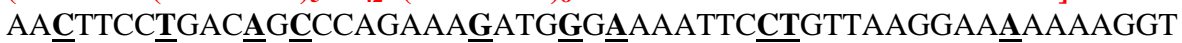

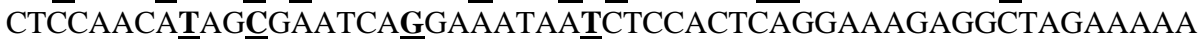
AGGGGACCCATCTA(1bp del)ATGGAGTCAGGGAAACA

H105 (GenBank Accession MH200595)

[269 bp del relative to allele 20 (N162+AGAGAT12+N35)]-

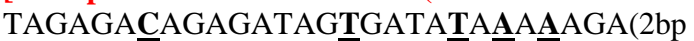

add)GATAGAGATAGAGATAGAGTTAGATATAGA(2bp add)GAGGT $\underline{\mathbf{T}}$ (4bp

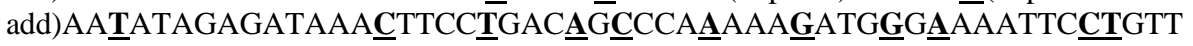

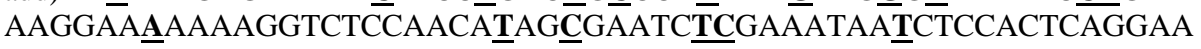

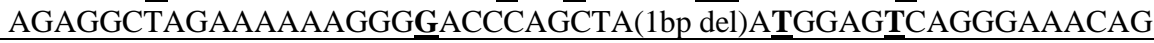

H-106 (GenBank Accession MH200596) 


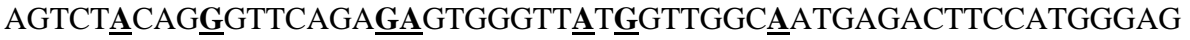

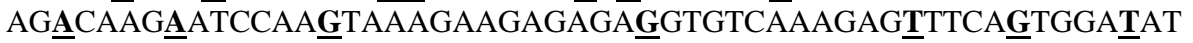

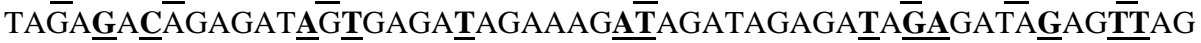

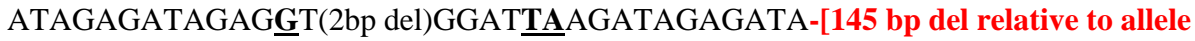
$20\left(\right.$ GAGAT+(AGAGAT) $5+\mathbf{N}_{42}+\left(\right.$ AGAGAT) $_{8}$ AGAGAGGTAAAGATAGAGAT]AACTTCCTGACAGCCCAGAAAGATGGGAAAATTCCTGTTAAGGAAAAAAAGGTC TCCAACATAGCGAATCAGGAAATAATCTCCACTCAGGAAAGA GGCTAGAAAAAAGGGGACCCAGCTA(1bp del)ATGGAGTCAGGGAAACAGACA 108 (Genbank Accession MH200597)

CAGGGTTCAGAAG(1bpdel)TGGGTTTTAGTTGGCTATGAGACTTCCAT $\underline{T} G G A G A G G$

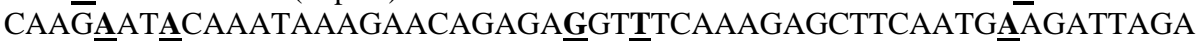

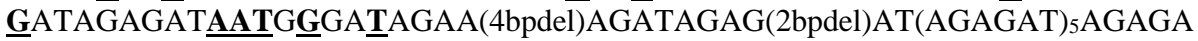

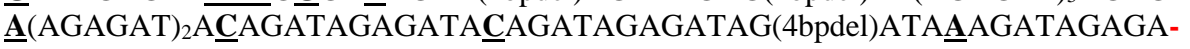
[96bp del relative to allele 20 (N29+ AGAGAT $_{8}$ )-AGAGAGGTAAAGATAGAGA]-

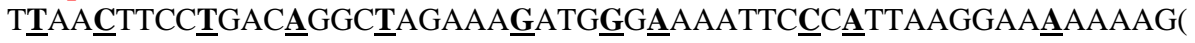

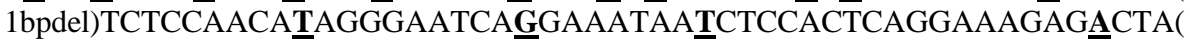
1bpdel)AAAAAAGGGAACCCAGCTA(1bpdel)ATGGAGTCAGGGAAACAG

\section{5 (Genbank Accession MH200598)}

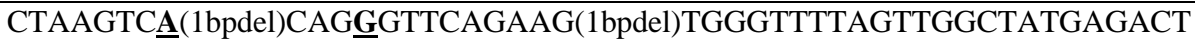

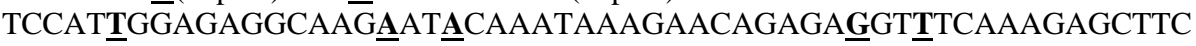

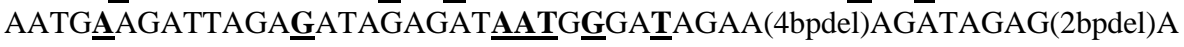

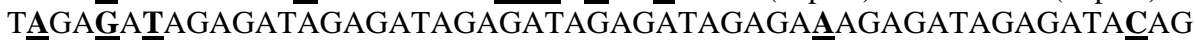

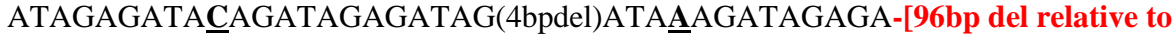
allele 20 (N29+ AGAGAT8)- AGAGAGGTAAAGATAGAGA]-

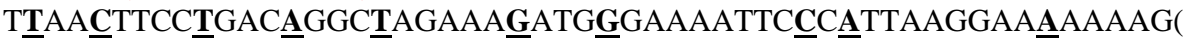
1bpdel)TCTCCAACA $\underline{\mathbf{T}}$ AGGGAATCA $\underline{\mathbf{G}}$ GAAATAATCTCCACTCAGGAAAGAG $\underline{\text { ACTA( }}$ 1bpdel)AAAAAAGGGAACCCAGCTĀ(1bpdel)ATGGAGTCAGGGAAACA

H-150 (GenBank Accession MH200599)

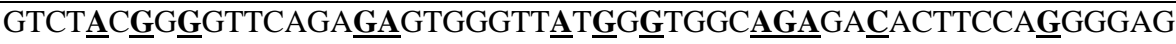

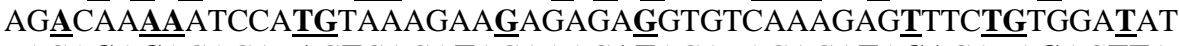

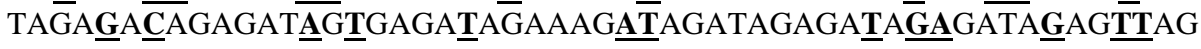

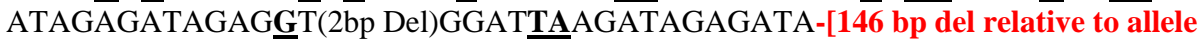
20 (GAGAT+(AGAGAT) $)_{5}+\mathbf{N}_{42}+(\text { AGAGAT) })_{8}$ AGAGAGGTAAAGATAGAGATA]

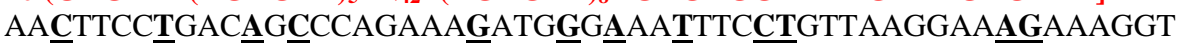

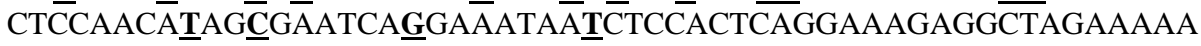
AGGGGACCCAGCTA(1bp del)ATGGAGTCAGGGAAACA

151 (Genbank Accession MH200600)

GCTAAGTC $\underline{\mathbf{A}}$ (1bpdel)CAGGGTTCAGAAG(1bpdel)TGGGTTTTAGTTGGCTATGAGAC

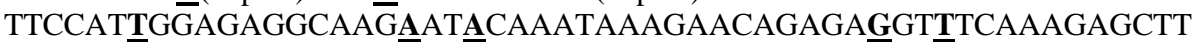

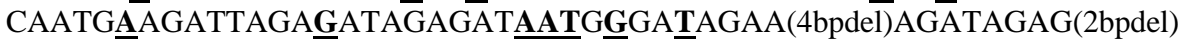

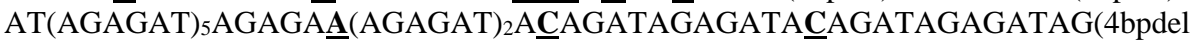
)ATA $\underline{A} A G A T A G A G A-[96 b p$ del relative to allele 20 (N29+ AGAGAT)AGAGAGGTAAAGATAGAGA]-

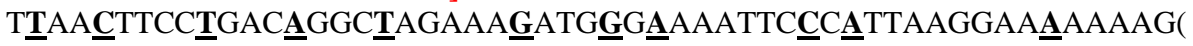
1bpdel)TCTCCAACA $\underline{\mathbf{T}}$ AGGGAATCA $\underline{\mathbf{G}}$ GAAATAATCTCCACTCAGGAAAGAG $\underline{\text { ACTA( }}$ 1bpdel)AAAAAAGGGAACCCAGCTĀ(1bpdel)ATGGAGTCCAGGGAAACAGG 


\begin{tabular}{|c|}
\hline H-152 (GenBank Accession MH200601) \\
\hline 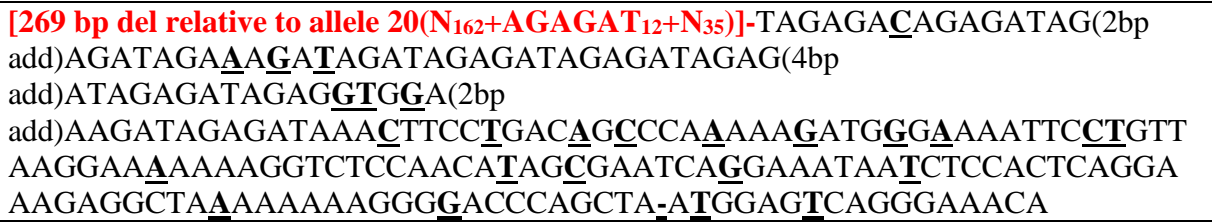 \\
\hline H-153 (Genbank Accession MH200602) \\
\hline 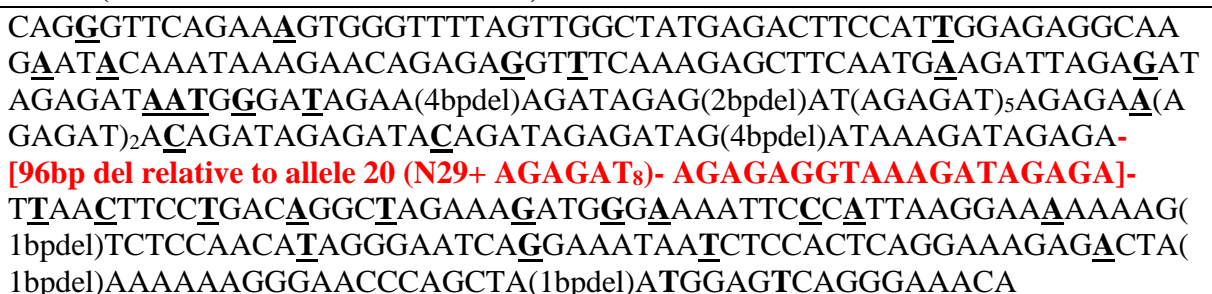 \\
\hline
\end{tabular}




\section{REFERENCE:}

[1] Qamar, R., Ayub, Q., Mohyuddin, A., Helgason, A., Mazhar, K., Mansoor, A., Zerjal, T., Tyler-Smith, C., Mehdi, S. Q., Am. J. Hum. Genet. 2002, 70, 1107-1124.

[2] Siddique, A., Afghanistan's Ethnic Divides, 2012.

[3] Zerjal, T., Xue, Y., Bertorelle, G., Wells, R. S., Bao, W., Zhu, S., Qamar, R., Ayub, Q., Mohyuddin, A., Fu, S., Li, P., Yuldasheva, N., Ruzibakiev, R., Xu, J., Shu, Q., Du, R., Yang, H., Hurles, M. E., Robinson, E., Gerelsaikhan, T., Dashnyam, B., Mehdi, S. Q., Tyler-Smith, C., Am. J. Hum. Genet. 2003, 72, 717-721.

[4] Adnan, A., Ralf, A., Rakha, A., Kousouri, N., Kayser, M., Forensic Sci. Int. Genet. 2016, $25,45-51$.

[5] Adnan, A., Rakha, A., Noor, A., Oven, M. van, Ralf, A., Kayser, M., Int. J. Legal Med. 2017.

[6] Adnan, A., Rakha, A., Lao, O., Kayser, M., Forensic Sci. Int. Genet. 2018.

[7] Jobling, M. A., Samara, V., Pandya, A., Fretwell, N., Bernasconi, B., Mitchell, R. J., Gerelsaikhan, T., Dashnyam, B., Sajantila, A., Salo, P. J., Nakahori, Y., Disteche, C. M., Thangaraj, K., Singh, L., Crawford, M. H., Tyler-Smith, C., Hum. Mol. Genet. 1996, 5, 1767-1775.

[8] Jobling, M. A., Lo, I. C. C., Turner, D. J., Bowden, G. R., Lee, A. C., Xue, Y., CarvalhoSilva, D., Hurles, M. E., Adams, S. M., Chang, Y. M., Kraaijenbrink, T., Henke, J., Guanti, G., McKeown, B., Oorschot, R. A. H. van, Mitchell, R. J., Knijff, P. de, TylerSmith, C., Parkin, E. J., Hum. Mol. Genet. 2007, 16, 307-316.

[9] Repping, S., Skaletsky, H., Brown, L., Daalen, S. K. M. van, Korver, C. M., Pyntikova, T., Kuroda-Kawaguchi, T., Vries, J. W. A. de, Oates, R. D., Silber, S., Veen, F. van der, Page, D. C., Rozen, S., Nat. Genet. 2003, 35, 247-251.

[10] Bosch, E., Jobling, M. A., Hum. Mol. Genet. 2003, 12, 341-347.

[11] Repping, S., Daalen, S. K. M. van, Brown, L. G., Korver, C. M., Lange, J., Marszalek, J. D., Pyntikova, T., Veen, F. van der, Skaletsky, H., Page, D. C., Rozen, S., Nat. Genet. 2006, 38, 463-467.

[12] Verma, R. S., Rodriguez, J., Dosik, H., J. Hered. 1982, 73, 236-238.

[13] Affara, N. A., Ferguson-Smith, M. A., Tolmie, J., Kwok, K., Mitchell, M., Jamieson, D., Cooke, A., Florentin, L., Nucleic Acids Res. 1986, 14, 5375-5387.

[14] Bernstein, R., Wadee, A., Rosendorff, J., Wessels, A., Jenkins, T., Hum. Genet. 1986, 74, 223-229.

[15] Page, D. C., Cold Spring Harb. Symp. Quant. Biol. 1986, 51 Pt 1, 229-235.

[16] Budowle, B., Aranda, X. G., Lagace, R. E., Hennessy, L. K., Planz, J. V., Rodriguez, M., Eisenberg, A. J., Int. J. Legal Med. 2008, 122, 421-427.

[17] Westen, A. A., Kraaijenbrink, T., Clarisse, L., Grol, L. J. W., Willemse, P., Zuniga, S. B., Robles de Medina, E. A., Schouten, R., Gaag, K. J. van der, Weiler, N. E. C., Kal, A. J., Kayser, M., Sijen, T., Knijff, P. de, Forensic Sci. Int. Genet. 2015, 14, 174-181.

[18] Balaresque, P., Bowden, G. R., Parkin, E. J., Omran, G. A., Heyer, E., Quintana-Murci, L., Roewer, L., Stoneking, M., Nasidze, I., Carvalho-Silva, D. R., Tyler-Smith, C., Knijff, P. de, Jobling, M. A., Hum. Mutat. 2008, 29, 1171-1180.

[19] Kayser, M., Roewer, L., Hedman, M., Henke, L., Henke, J., Brauer, S., Krüger, C., Krawczak, M., Nagy, M., Dobosz, T., Szibor, R., Knijff, P. de, Stoneking, M., Sajantila, A., Am. J. Hum. Genet. 2000, 66, 1580-1588. 
[20] Larmuseau, M. H. D., Vanderheyden, N., Van Geystelen, A., Decorte, R., Forensic Sci. Int. Genet. 2014, 11, 214-219.

[21] Davis, C., Ge, J., Sprecher, C., Chidambaram, A., Thompson, J., Ewing, M., Fulmer, P., Rabbach, D., Storts, D., Budowle, B., Forensic Sci. Int. Genet. 2013, 7, $204-208$.

[22] Consortium, T. Y. C., Genome Res. 2002, 12, 339-348.

[23] Prinz, M., Boll, K., Baum, H., Shaler, B., Forensic Sci. Int. 1997, 85, 209-218.

[24] Zhang, S., Tian, H., Wang, Z., Zhao, S., Hu, Z., Li, C., Ji, C., Forensic Sci. Int. Genet. 2014, 13, 112-120.

[25] Nei, M., Molecular Evolutionary Genetics, Columbia University Press, New York, 1997.

[26] Kumar, S., Stecher, G., Tamura, K., Mol. Biol. Evol. 2016, 33, 1870-1874.

[27] R Core Team, $R$ : A language and environment for statistical computing, R Foundation for Statistical Computing, Vienna, Austria., 2015.

[28] Quintana-Murci, L., Chaix, R., Wells, R. S., Behar, D. M., Sayar, H., Scozzari, R., Rengo, C., Al-Zahery, N., Semino, O., Santachiara-Benerecetti, A. S., Coppa, A., Ayub, Q., Mohyuddin, A., Tyler-Smith, C., Qasim Mehdi, S., Torroni, A., McElreavey, K., Am. J. Hum. Genet. 2004, 74, 827-845.

[29] Haber, M., Platt, D. E., Ashrafian Bonab, M., Youhanna, S. C., Soria-Hernanz, D. F., Martínez-Cruz, B., Douaihy, B., Ghassibe-Sabbagh, M., Rafatpanah, H., Ghanbari, M., Whale, J., Balanovsky, O., Wells, R. S., Comas, D., Tyler-Smith, C., Zalloua, P. A., Genographic Consortium, PloS One 2012, 7, e34288.

[30] Dulik, M. C., Osipova, L. P., Schurr, T. G., PLoS ONE 2011, 6, e17548.

[31] Derenko, M., Malyarchuk, B., Grzybowski, T., Denisova, G., Dambueva, I., Perkova, M., Dorzhu, C., Luzina, F., Lee, H. K., Vanecek, T., Villems, R., Zakharov, I., Am. J. Hum. Genet. 2007, 81, 1025-1041.

[32] Rakha, A., Fatima, Peng, M.-S., Adan, A., Bi, R., Yasmin, M., Yao, Y.-G., Forensic Sci. Int. Genet. 2017, 30, e1-e5.

[33] Chang, C.-W., Mulero, J. J., Budowle, B., Calandro, L. M., Hennessy, L. K., J. Forensic Sci. 2006, 51, 344-348.

[34] Collins, F. S., Brooks, L. D., Chakravarti, A., Genome Res. 1998, 8, 1229-1231.

[35] Fredman, D., Nucleic Acids Res. 2004, 32, 516D - 519.

[36] NCBI Resource Coordinators, Agarwala, R., Barrett, T., Beck, J., Benson, D. A., Bollin, C., Bolton, E., Bourexis, D., Brister, J. R., Bryant, S. H., Canese, K., Cavanaugh, M. Charowhas, C., Clark, K., Dondoshansky, I., Feolo, M., Fitzpatrick, L., Funk, K., Geer, L. Y., Gorelenkov, V., Graeff, A., Hlavina, W., Holmes, B., Johnson, M., Kattman, B., Khotomlianski, V., Kimchi, A., Kimelman, M., Kimura, M., Kitts, P., Klimke, W., Kotliarov, A., Krasnov, S., Kuznetsov, A., Landrum, M. J., Landsman, D., Lathrop, S., Lee, J. M., Leubsdorf, C., Lu, Z., Madden, T. L., Marchler-Bauer, A., Malheiro, A., Meric, P., Karsch-Mizrachi, I., Mnev, A., Murphy, T., Orris, R., Ostell, J., O’Sullivan, C., Palanigobu, V., Panchenko, A. R., Phan, L., Pierov, B., Pruitt, K. D., Rodarmer, K., Sayers, E. W., Schneider, V., Schoch, C. L., Schuler, G. D., Sherry, S. T., Siyan, K., Soboleva, A., Soussov, V., Starchenko, G., Tatusova, T. A., Thibaud-Nissen, F., Todorov, K., Trawick, B. W., Vakatov, D., Ward, M., Yaschenko, E., Zasypkin, A., Zbicz, K., Nucleic Acids Res. 2018, 46, D8-D13.

[37] Chang, Y. M., Perumal, R., Keat, P. Y., Kuehn, D. L. C., Forensic Sci. Int. 2007, 167, 7076. 
[38] Park, M. J., Shin, K.-J., Kim, N. Y., Yang, W. I., Cho, S.-H., Lee, H. Y., J. Forensic Sci. 2008, 53, 331-334.

[39] Parkin, E. J., Kraayenbrink, T., Opgenort, J. R. M. L., Driem, G. L. van, Tuladhar, N. M., Knijff, P. de, Jobling, M. A., Forensic Sci. Int. 2007, 166, 176-181.

[40] Mizuno, N., Nakahara, H., Sekiguchi, K., Yoshida, K., Nakano, M., Kasai, K., Forensic Sci. Int. 2008, 174, 71-76.

[41] Roewer, L., Krüger, C., Willuweit, S., Nagy, M., Rodig, H., Kokshunova, L., Rothämel, T., Kravchenko, S., Jobling, M. A., Stoneking, M., Nasidze, I., Forensic Sci. Int. 2007, 173, 204-209.

[42] Sánchez, C., Barrot, C., Xifró, A., Ortega, M., Aranda, I. G. de, Huguet, E., Corbella, J., Gené, M., Forensic Sci. Int. 2007, 172, 211-217.

[43] Tarlykov, P. V., Zholdybayeva, E. V., Akilzhanova, A. R., Nurkina, Z. M., Sabitov, Z. M., Rakhypbekov, T. K., Ramanculov, E. M., Croat. Med. J. 2013, 54, 17-24. 